THE EFFECT OF BINGE-LIKE ETHANOL EXPOSURE ON ADOLESCENT PERFORMANCE IN NON-SPATIAL AND SPATIAL FORMS OF THE NOVEL OBJECT RECOGNITION PARADIGM

BY

\title{
MIRIAM COLLINS
}

\author{
A thesis \\ submitted to the Victoria University of Wellington \\ in fulfilment of the requirements for the degree of \\ Master of Science in Cognitive and Behavioural \\ Neuroscience
}

Victoria University of Wellington

2016 
Table of contents

Page

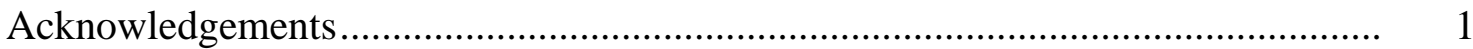

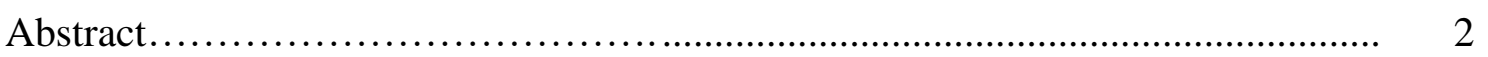

List of figures and tables....................................................................... 3

Introduction............................................................. 5

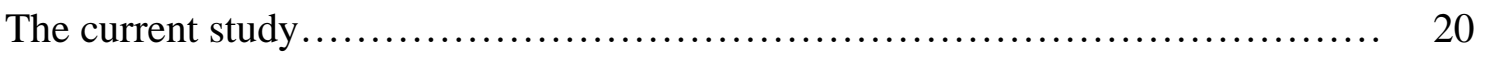

Method..................................................................... 21

Results and discussion.............................................. 25

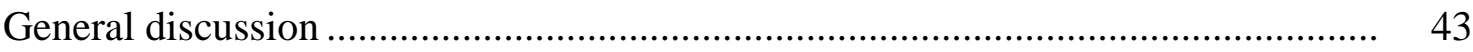

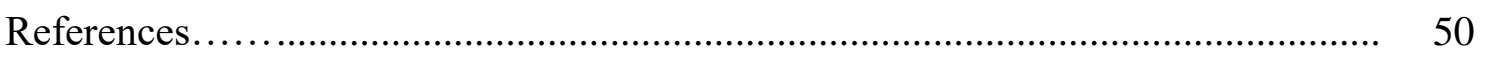


Acknowledgements

This thesis is dedicated to my dad. I would like to thank my supervisor Susan Schenk for her guidance throughout my MSc, as well as Joyce Colussi-Mas, Sophie Muller, and Michael Roberts for their help with the technical side of things. I also want to thank all the people I did CBNS with for enrolling in the course at the same time as me and making the last two years a bit more colourful and exciting. 


\begin{abstract}
Previous research has demonstrated that ethanol produces differential effects on non-spatial or recognition memory and spatial memory; spatial memory deficits were consistently found to be more persistent than non-spatial memory deficits. Ethanol-produced deficits have also been found to be dependent on age at exposure, and exposure during adolescence produced more persistent deficits than when exposure was experienced by older subjects.

The current study investigated the effects of a 'binge-like' 5 day episode of ethanol exposure $(1.0 \mathrm{~g} / \mathrm{kg} \times 5)$ on performance in non-spatial and spatial forms of the novel object recognition (NOR) task. Subjects were exposed either during adolescence or following maturity. Tests were conducted 2 or 9 days following exposure. NOR was tested following inter-trial intervals of 1,3 , or 5 minutes. Data from mature rats could not be obtained or analysed due to procedural issues that precluded NOR measurement. Control rats failed to demonstrate NOR at any of the time intervals. Reasons for these negative findings are discussed.
\end{abstract}


List of figures and tables

Table 1. Cycle 1 Novel Object Recognition (NOR) task items.

Table 2. Cycle 2 Novel Object Recognition (NOR) task items.

Figure 1. Time spent on the old rota-rod following daily administration of saline (0.9\%, IP) or ethanol $(1 \mathrm{~g} / \mathrm{kg}, \mathrm{IP})$ at time zero, twenty, and forty.

Table 3. Analysis of variance following acute administration of saline or ethanol on initial rota-rod apparatus performance.

Figure 2. Time spent on the second rota-rod apparatus following daily administration of saline $(0.9 \%$, IP) or ethanol $(1 \mathrm{~g} / \mathrm{kg}, \mathrm{IP})$ at time zero, twenty, and forty.

Table 4. Analysis of variance of rota-rod performance following acute administration of saline or ethanol.

Figure 3. Number of approaches to each object in T1 (1) and T2 (2) for saline (A) and ethanol (B) treated animals across each cycle 1 non-spatial novel objection recognition trial. Figure 4. Proportion of number of approaches to duplicate objects in T1 and replacement novel objects in T2 for saline (A) and ethanol (B) treated animals across each cycle 1 nonspatial novel objection recognition trial.

Figure 5. Time spent exploring both objects in T1 (1) and T2 (2) for saline (A) and ethanol (B) treated animals during the cycle 1 non-spatial objection recognition task.

Figure 6. Proportion of time spent exploring duplicate objects in T1 and replacement novel objects in T2 for saline (A) and ethanol (B) treated animals during the cycle 1 non-spatial novel objection recognition task. 
Figure 7. Number of approaches to each object in T1 (1) and T2 (2) for saline (A) and ethanol (B) treated animals across each cycle 1 spatial novel objection recognition trial.

Figure 8. Proportion of number of approaches to duplicate objects in T1 and replacement novel objects in $\mathrm{T} 2$ for saline (A) and ethanol (B) treated animals across each cycle 1 spatial novel objection recognition trial.

Figure 9. Time spent exploring both objects in T1 (1) and T2 (2) for saline (A) and ethanol (B) treated animals during each trial of the cycle 1 spatial novel objection recognition task.

Figure 10. Proportion of time spent exploring duplicate objects in $\mathrm{T} 1$ and replacement novel objects in T2 for saline (A) and ethanol (B) treated animals during the cycle 1 spatial novel objection recognition task.

Table 5. Significant effects obtained from analysis of various measures of performance in Cycle 2 non-spatial NOR trials.

Table 6. Significant effects obtained from analysis of various measures of performance in Cycle 2 spatial NOR trials. 
Alcohol is one of the most commonly abused drugs in the world (Centers for Disease Control and Prevention, 2014), and this abuse contributes to approximately $1 / 10$ deaths in those aged 20-64, and 3.3 million deaths worldwide each year (World Health Organisation, 2015). Harmful use of alcohol can also play a causal role in over 200 diseases, such as mental illness (or the exacerbation of), heart disease, cancer, anaemia, liver cirrhosis, and pancreatitis, as well as various memory deficits (CDC, 2014; WHO, 2015).

A binge drinking episode is defined as the consumption of a large quantity of alcohol in one session in order to become intoxicated. This is approximately $5+$ drinks for men, and 4+ drinks for women (Foundation for a Drug-Free World, 2015). Multiple binge drinking episodes separated by withdrawal periods is defined as binge drinking in general, or chronic intermittent exposure (Hiller-Sturmhöfel and Swartzwelder, 2004).

Binge drinking is a common activity in multiple western countries (Hiller-Sturmhöfel and Swartzwelder, 2004). In New Zealand adolescents on average have their first drink at 14.5 years of age, with $45 \%$ of secondary school students considering themselves to be regular drinkers, and $18 \%$ classifying as binge drinkers who drink to excess at least $3+$ times a week (Adolescent Health Research Group, 2012). Adolescent binge drinking is also prevalent in the U.S.A., where underage drinkers consume more alcohol on average per drinking session than adults, and those aged 12-20 are responsible for the consumption of $11 \%$ of all alcohol consumed within the nation (Centers for Disease Control and Prevention, 2015). These figures are of concern as binge drinking can result in both short- and long-term impairments in cognition and memory due to persistent effects on the brain (HillerSturmhöfel and Swartzwelder, 2004).

This susceptibility of the human brain to the effects of alcohol abuse can differ depending on factors such as age and the developmental period of the brain. Adolescence is 
one of the most critical periods of brain development. During this time there are alterations in neurochemistry, an increase in gray and white matter throughout the brain, increased development of different areas (e.g. prefrontal cortex) and development of circuity between different regions (e.g. fronto-subcortical circuitry development (Giedd, 2006). These processes can be interrupted by alcohol exposure, resulting in later life dysfunction of executive control, and disruption in the encoding, storage, and retrieval of memories due to shrinkage of the responsible areas such as the prefrontal cortex and hippocampus (HillerSturmhöfel and Swartzwelder, 2004).

At the other end of the developmental spectrum, alcohol consumption by retirees is also increasing (Institute of Alcohol Studies, 2016). The drastic change of post-employment life can result in increased alcohol consumption (Institute of Alcohol Studies, 2016), particularly if the individual was forced into retirement rather than choosing it for themselves (Gallo, Bradley, Siegel, and Kasl, 2001). Three types of elderly drinkers have been identified: early-onset drinkers, who have a continuing drinking problem that developed earlier in their lives, late-onset drinkers, who start drinking later in life, typically in response to negative experiences such as bereavement or retirement, and intermittent types, who drink to excess occasionally (Institute of Alcohol Studies, 2016).

A longitudinal study on a group of mostly older-onset problem drinkers who had been assessed for treatment found that all subjects reported drinking most commonly $4+$ drinks on a daily basis, similar to binge levels seen in adolescents (Christie, Bamber, Powell, Arrindell, and Pant, 2012). More research in this area is required to adequately address or the effect of later-onset drinking, as not much is known about the detrimental effects of alcohol on the elderly brain.

\section{The hippocampus and spatial memory}


Alcohol-related memory impairments may relate to drug-produced changes in the hippocampus (Ryabinin, 1998). Human studies have shown that those diagnosed with adolescent-onset alcohol use disorders have significantly smaller right and left hippocampal volumes compared to age-matched controls. These affects were negatively correlated with the disorder duration (De Bellis et al., 2000). On subjects with older onset alcoholism females demonstrated smaller bilateral hippocampus volumes, but males only had a significantly smaller right hippocampus (Agartz, Momenan, Rawlings, Kerich, and Hommer, 1999). Postmortem analysis of human brain tissue also demonstrates that alcoholism results in a loss of white matter (Jensen and Pakkenberg, 1993), and decreased cortical gray matter density (Kril, Halliday, Svoboda, and Cartwright, 1997).

On rodents both pre-natal (Barnes and Walker, 1981) and long-term chronic postnatal exposure (Walker, Barnes, Zornetzer, Hunter, and Kubanis, 1980) to ethanol results in a permanent reduction of CA1 and CA3 hippocampal pyramidal cells. A question remains as to whether these brain deficits are reflected in behavioural deficits.

Spatial memory encodes information about spatial orientation within the surrounding environment (Burgess, Maguire, and O'Keefe, 2002) This type of memory permits navigation of familiar areas such as the route you take to drive to work (O'Keefe and Nadel, 1978), or in the case of animal studies the location of the food that was previously stored (Bird, Roberts, Abroms, Kit, and Cupri, 2003). This encoded spatial information can be stored as working, short and long term memories (Burgess, Maguire, and O'Keefe, 2002), and it is known that the hippocampus is involved in these processes (Burgess, Maguire, and O'Keefe, 2002).

The hippocampus plays a role as the "cognitive map" of the brain, and the pyramidal hippocampal cells are the basic units of this map. These cells internally represent specific physical points in space and allow for referencing of the surrounding environment and ease of 
navigation (O'Keefe and Nadel, 1978). Hippocampal units differentially fire depending on the relative position of subjects within an environment (Best and Ranck, 1975; Hill, 1978; O'Keefe and Dostrovsky, 1971; Olton, Walker, and Gage, 1978; Thompson and Best, 1990).

Human research assessing the effects of ethanol on spatial and non-spatial (recognition) memory

Due to ethical constraints around the administration of alcohol to human subjects, alcoholism recovery status, and increased risk of injuries (NIAAA, 2015), research involving the effect of alcohol on memory in humans is often limited to studies with participants who already have an alcohol-related disorder.

Studies often employ the Spatial Working Memory (SWM) task that presents the participant with a number of boxes on the screen which are opened by a process of elimination to locate tokens. Adolescents of both genders diagnosed with alcohol use disorder show a diminished blood oxygen level-dependent (BOLD) response on fMRI scans in the left precentral gyrus and bilateral cerebellar areas during the SWM task but performance on this task was not compromisd. These abnormalities in brain response were particularly enhanced in subjects who reported higher consumption levels of alcohol and/or hangover symptoms (Tapert et al., 2004). Those who qualified as "binge drinkers" (as defined by scores from the Alcohol Use Questionnaire) consistently displayed between-search errors in the Spatial Working Memory task (Townshend and Duka, 2005; Weissenborn and Duka, 2003; Scaife and Duka, 2009).

Recognition memory comes under the umbrella of declarative or explicit memory, and refers to the ability to recall previous events, objects, and people encountered on a previous occasion. A second presentation of the stimulus is necessary in order for the subject to determine whether it is familiar, and does not require memory of the actual episode in 
which the initial presentation occurred. This is unlike recall memory where the subject freely describes a previous encounter without any external stimuli to prompt them (Manns, Hopkins, Reed, Kitchener, and Squire, 2003).

FAS children display a deficit in object recognition memory compared to agematched controls when a delay is implemented, but not when recall is immediate (Uecker and Nadel, 1995). A case study on an amnesic individual with focal perinatal hippocampal damage revealed that when the subject was presented with a virtual town square scene and had to later recall object locations within the image from the same viewpoint, accuracy was similar to controls. However, when the subject had to recall object locations within the scene from a new viewpoint, performance was significantly impaired in contrast to controls (King, Trinkler, Hartley, Vargha-Khadem and Burgess, 2004). This indicates that different underlying processes are involved in recognition memory and memory involving spatial navigation.

Acute alcohol exposure has also been shown to have an effect on forced-choice picture recognition task performance in subjects who have not previously been diagnosed with an alcohol-related disorder. In a study by Parker, Birnbaum, and Noble (1976), subjects received either no alcohol, or a medium $(0.5 \mathrm{mg} / \mathrm{kg})$ or high $(1 \mathrm{mg} / \mathrm{kg})$ amount immediately prior to engaging in the task. Subjects were presented 40 images, at 4 second intervals. Following this the familiar images were paired and presented with similar but novel distractor images, and subjects were asked to choose which of the images they had seen before. The results revealed a forgetting curve, with those who received the highest dose of alcohol correctly recognising the least amount of previously viewed images, followed by the medium dose group, and the group that received no alcohol. 
Studies with humans are limited due to ethical constraints, including the administration of an addictive substance, the potential danger of a subject hurting oneself when intoxicated due to weakened psychomotor functioning or inappropriate or dangerous behaviour due to lowered inhibitions (Tabakoff and Hoffman, 2000).

\section{The benefit of using animal models in alcohol research}

In animal models you can mimic both acute, chronic, and intermittent ethanol administration, while controlling for the one aspect of alcohol related behaviour you are interested in studying (such as memory functioning after a "binge-like" episode of drinking). This is done by minimising confounding variables, something which cannot be easily done in human research as you can never be certain about what subjects are doing outside of the time you see them. Researchers can also control both health and past substance use, important things which can inexplicably effect results when not mentioned or taken into consideration (Tabakoff and Hoffman, 2000). Rodents have similar biochemical and physiological systems to humans, so results produced by rodent models can be considered to have predictive validity, where by ethanol administration can be said to predict the same outcome in humans who have received an equivalent amount of the substance in an equivalent time period (e.g. intoxication, tolerance, dependence, organ damage). (Tabakoff and Hoffman, 2000).

Past substance use can be controlled in animal models. This ensures that a change in cognition or behaviour is due to a treatment received within a study and not due to extraneous factors, something which could occur in a study with humans where a subject is not completely honest about their past or present medical history and/or substance use (Tabakoff and Hoffman, 2000).

This is particularly beneficial for studying the effects of ethanol on cognitive and behavioural processes across ages. Though anatomical and physiological differences must be 
taken into consideration, there are approximate equivalencies in ages that can be determined (Andreollo, Santos, Araujo, and Lopes, 2012). One month in the rat is approximately equal to 3 human years, with rats reaching the equivalence of 18 years at around 6 months of age. Earlier adolescence occurs at around 2-3 months, and 2.5 rat years equates to 65 human years (Andreollo et al., 2012).

\section{Common paradigms used to assess spatial and non-spatial or recognition memory}

Numerous paradigms exist to measure different aspects of spatial memory in animals. The Morris Water Maze Task (MWMT) was originally designed to assess spatial working memory in particular, but has been adapted to also examine encoding and retrieval of short and long term spatial representations and recognition memory. In this paradigm a rodent is placed in an open field maze filled with opaque water with a hidden escape platform. The most common dependent variable measured is latency to escape the water maze. This can be measured across training sessions to analyse retention, depending on the amount of sessions and the time in between them (Morris, 1984). Successful performance in the MWMT is reliant on intact cognitive mapping abilities (Morris, 1984).

Rats with hippocampal lesions displayed significantly poorer performance in the MWMT, as shown by an increase in latency to find the hidden platform, and a longer total distance travelled in the water. This deficit was persistent, and was still apparent following 28 trials (Morris, Garrud, Rawlins, and O'Keefe, 1982). Guinea pigs prenatally exposed to ethanol showed similar deficits (Richardson, Byrnes, Brien, Reynolds, Dringenberg, 2002).

The MWMT has been modified so that it is a delayed matching to place (DMP) task, in that the subject must learn to locate a constantly changing hidden platform in opaque water. The task begins with a search trial where the animal discovers the hidden platform, followed by an inter-trial interval (ITI) or delay of alternating length and a testing phase 
where the subject must remember the location of the hidden platform in order to escape. This assesses short-term spatial memory, and has been proved effective in revealing deficits produced by both hippocampal lesions (Steele and Morris, 1999) and ethanol exposure (Clements, Girard, Ellard, and Wainwright, 2005). However this version of the task requires at least one week of training (Steele and Morris, 1999).

Object recognition memory specifically refers to the ability to differentiate between novel objects and familiar objects encountered on a previous occasion. Rodents can recognise objects through both visual, tactile, and olfactory cues (Manns and Eichenbaum, 2009).This form of memory is often assessed in animals using the delayed non-matching to samples (DNMS) task. Much like the DMP task, The DNMS task involves the presentation of a stimulus followed by an ITI and presentation of the old stimulus paired with a novel stimulus. Subjects must visibly select the novel stimulus in the second presentation with many hours of training being required to reach criterion performance (Chudasama, 2010).

The novel object recognition paradigm also assesses object recognition memory through presentation of familiar and novel stimuli. It is a one-trial task involving a familiarisation phase at time 1 (T1), during which the subject is placed in a chamber with two identical objects. The subject is then removed and one object is switched for a novel item or in the case of the spatial adaptation, one of the identical objects is moved to a new location within the arena. A delay or inter-trial interval (ITI) is then imposed, before the subject is returned to the chamber for the test phase at Time 2 (T2). The dependent measure is the amount of exploration of each object, which is recorded as both the amount of approaches and time spent with each object (Antunes and Biala, 2012).

The primary purpose of the NOR task is to assess recognition memory after different delay times between $\mathrm{T} 1$ and $\mathrm{T} 2$ have been imposed. This paradigm is a useful and efficient 
tool for testing memory as it requires no training. However, several aspects of the procedure have implications for unambiguous interpretation of the data. The habituation phase allows the subject to become familiar with the novel testing environment itself, reducing anxiety levels during the actual experimental trials and ensuring that the subjects will explore the objects rather than the environment itself (Silvers, Harrod, Mactutus, and Booze, 2007). It is important, therefore, to ensure a long enough habituation period.

It is critical for subjects to explore both objects for a criterion amount of time during T1. Because some rats fail to explore, their data cannot be interpreted and so cannot be used. As a result, sample sizes can be substantially smaller than what one initially starts with. The minimum criterion for exploration time in the familiarisation phase varies widely between studies, with total exploration times as small as 10 seconds or more appearing sufficient enough to allow measurement of encoding and retention (Camarasa, Marimon, Rodrigo, Escubedo, and Pubil, 2008). Required times of up to 20 minutes have also been reported (Goulart et al., 2010).

The NOR paradigm relies on the natural preference to explore novel items (Antunes and Biala, 2012). In this respect, it does not depend on reinforcers, as do most other memory tasks. The procedure however is more taxing on cognitive load in comparison to tasks where the subject explores a single novel object or novel environment, as the subject must maintain the two identical T1 objects in their working memory in order to later discriminate between the familiar and novel objects in T2 (Silvers et al., 2007).

A spatially modified version of this task that investigates novel location recognition memory also exists (Ennaceur and Meliani, 1992). This uses the same methodology in T1, with two identical objects being placed equidistantly apart from each other and the back wall of the testing arena, but in T2 both objects are placed back in the arena, with the sample 
object in the same location as previously, and the duplicate object placed in a novel location (Ennaceur and Meliani, 1992).

In both procedures memory is inferred when the subject explores the novel object/location more than the familiar object/location, due to rodents having an innate preference for novelty (Antunes and Biala, 2012). This preference for novelty occurs when memory of the familiar object/placement is easily accessible, shortly after exposure to the objects has occurred. Amount of contact with a novel object/placement is dependent on memory of the familiar object, thus allowing the subject to discriminate between the two objects. When the memory is in an intermediate phase and not as readily accessible both objects are explored equally, while if the memory is in a remote phase it is more difficult to access, resulting in forgetting of which object the subject has previously exposed to. The transition between these memory phases results in a forgetting curve, with the amount of contact with the novel object/placement decaying as the ITI time increases. (Antunes and Biala, 2012).

There are several ways to measure and identify novel object recognition. The original measure as devised by Ennaceur and Delacour (1988) was time spent exploring both the novel and familiar objects in T2, with a longer length of time spent exploring the novel object being an indicator that the subject recognised the familiar object from previous encounters.

Ennaceur (2010) later argued that the frequency of approaches to each object was a more appropriate measure of recognition memory, being more sensitive to both strain and gender differences. Another measure is the recognition index, which can be a proportion of either the time spent exploring or approaches made to the novel object in T2, relative to total T2 exploration time or approaches (Antunes and Biala, 2012). This can be beneficial measure when there is a high amount of between subject variability in the amount of exploration time 
or approaches made, as it allows the researcher to get a clearer picture of where the true preference lies across ITIs and groups.

The success of this differentiation between familiar and novel can be dependent on variables that alter memory encoding, consolidation, and/or retrieval, such as brain lesions or pharmacological substances (Antunes and Biala, 2012). Previous research shows that hippocampal lesions impair performance for object memory in the NOR paradigm. Broadbent, Gaskin, Squire, and Clark (2010) investigated the effect of hippocampal lesions on NOR task performance. All subjects were allowed 12 five minute exploration sessions with two familiar objects followed by bilateral hippocampal lesions either 1 day, 4 weeks, or 8 weeks after the final exploration session. Two weeks post-surgery all subjects underwent a retention test. Both the 1 day and 4 week lesion groups displayed impaired object recognition memory, whereas the 8 week lesion group performed similarly to controls, displaying a novel object preference, suggesting that consolidation of memory had occurred.

Studies assessing the effects of MDMA (Schenk, Harper, and Do, 2011), methamphetamine (Schröder, O'Dell, and Marshall, 2003), cocaine (Schindler, Li, and Chavkin, 2010), and ketamine (Goulart et al., 2010) on NOR performance also show that the negative impact drugs of abuse have on memory can be successfully measured in this paradigm.

A meta-analysis of papers that investigated the role of the hippocampus in NOR performance, indicated that a forgetting effect is most consistently found following delays of 10 minutes (Cohen and Stackman, 2015). Normal rats aged post-natal day (PND) 29 and above displayed typical recognition memory profiles in the NOR paradigm following delays of up to 24 hours, but not following a delay of 48 hours (Reger, Hovda, and Giza, 2009). 
There is limited research on the effect of ethanol on NOR paradigm performance, but one study by Stragier et al. (2015) found that C57BL/6J mice exposed to a chronic freechoice ethanol treatment for 21 days impaired NOR performance after both a 1 hour and 24 hour delay, an effect which was particularly pronounced after the 24 hour delay. However it is important to note that ITIs shorter than an hour in length were not included in this study. This might be critical since other studies (Schenk et al. 2011), have found MDMA caused memory deficits that are observed following shorter ITIs.

The authors of the original novel object recognition paradigm paper have stated that a minimum time of 20 seconds exploration during T1 is adequate (Ennaceur and Delacour, 1988). Even if subjects meet or exceed the minimum exploration time, they can also be eliminated from further analyses if they fail to explore both objects in the testing phase, because if only one object is explored for the duration of the second phase it cannot truly be determined whether it is due to preference or another extraneous variable (Ennaceur and Delacour, 1988).

\section{Animal research assessing the effects of ethanol on spatial and non-spatial (recognition) memory}

A wide range of studies have linked both acute and chronic alcohol exposure to impairments in spatial memory in animals. There is evidence that both ethanol exposure and hippocampal damage also have a detrimental effect on object recognition memory, but contradictory findings exist as well. A dose-dependent memory impairment, as measured by NOR, was demonstrated in mice exposed to ethanol depending on when the ethanol was administered (Ryanbinin, Miller, and Durrant, 2002). Additionally, most subjects that were administered saline or the lower dose of ethanol spent more time exploring the novel object, whereas the majority of subjects that received the high ethanol dose spent a similar amount of 
time with both objects. The interpretation of the results is difficult, however, because there was no habituation phase.

Adolescent and adult rats given an aqueous ethanol solution for their sole source of fluid starting from PND 21 until either PND 90 or PND 180 showed significantly impaired DNMS performance when tested following a delay of an hour (but not delays of 1 or 15 minutes) (García-Moreno et al., 2002). Similar DNMS results were produced in monkeys with hippocampal lesions when delays were longer than 8 seconds (Zola et al., 2000), but no deficits were produced in monkeys with neonatal hippocampal lesions (Pascalis and Bachevalier, 1999).

Animal models of FAS have also been shown to result in spatial memory deficits in later life. Matthews and Simson (1998) found that adult rats prenatally exposed to ethanol demonstrated longer latencies to find the platform in the spatial version of the MWMT, but only when a 3-day delay between training and testing was imposed rather than a 1 day delay. The same effect has also been found when tested without a delay (Kim et al., 1997). Rats that were prenatally exposed to ethanol were tested first in the DNMS followed by the spatial version of the MWMT. No significant difference between groups was found for DNMS performance, but for the MWMT prenatally exposed rats took significantly longer to learn find the submerged platform across all 10 daily sessions.

Similar results have been found in animal studies with exposure to ethanol in adolescence and adulthood. Acute ethanol exposure greater than or equal to $1.5 \mathrm{~g} / \mathrm{kg}$ resulted in longer latencies for locating the submerged platform in the spatial version of the MWMT for both age groups. However, in the non-spatial version of the paradigm, no significant differences were found between groups, demonstrating a dissociation in the effect of ethanol exposure on the two types of memory (Matthews, Morrow, Tokunaga, and McDaniel, 2002) 
Similar patterns of performance in the spatial version of the MWMT were produced in mice that received ethanol $(1.75$ or $2.25 \mathrm{~g} / \mathrm{kg})$. There was no difference for the non-spatial version of the task (Berry and Matthews, 2004).

Adult rats were given either a vehicle or binge alcohol treatment followed by additional vehicle or ethanol doses for four consecutive days. Rats were tested five days (after ethanol withdrawal symptoms ceased), and ten weeks following this exposure. Within ten consecutive sessions (at both testing periods), rats underwent an object exploration task (OET) for both spatial memory and object recognition. The treatment produced an impairment in detecting spatial change. The ethanol treatment produced deficits in the nonspatial memory OET sessions when tested five days after exposure, but not at 10 weeks postexposure (Cippitelli et al., 2010).

The impact of both acute and chronic ethanol exposure on spatial and non-spatial memory was further compared in adult rats from PND 21-90 (García-Moreno and Cimadevilla, 2012). Those in the chronic exposure group received free access to an aqueous ethanol solution as their only liquid source, while those in the acute exposure group received one $2 \mathrm{~g} / \mathrm{kg}$ injection (I.P) per week. Chronic ethanol treatment produced deficits in both nonspatial and spatial versions of the DNMS task. In the non-spatial DNMS task both ethanol treated groups displayed similar levels of novel object preference compared to the control subjects following delays of 1 and 15 minutes, but performance was significantly worse following a 60 minute delay. In the spatial DNMS task both ethanol treated groups displayed memory deficits following all delays imposed. However, the acute ethanol treatment group still outperformed the chronic ethanol treatment group at the 1 and 15 minute delays. 
In summary, spatial memory impairments are more consistently present after both acute and chronic ethanol exposure, and are more persistent than non-spatial memory impairments.

\section{Effects of ethanol on memory as a function of age}

Numerous studies indicate that alcohol causes more severe memory impairments in spatial memory domains relative to object memory. However, it appears that the severity of this spatial memory impairment is age dependent. Several studies have found that adolescent rats were more sensitive than adults to acute ethanol exposure prior to MWMT and sand box maze task testing (Markwiese, Acheson, Levin, Wilson, \& Swartzwelder, 1998; Obernier et al., 2002; Rajendran and Spear, 2004), and with chronic exposure adolescent rats displayed an increased suppression of hippocampal long term potentiation (Swartzwelder, Wilson, and Tayyeb, 1995; Pyapali, Turner, Wilson, and Swartzwelder, 1999). Adolescent rats also took longer to recover from spatial memory deficits produced by long-term ethanol exposure (Sircar and Sircar, 2005; Rajendran and Spear, 2004).

Adult and aged C57Bl/6 mice provided with ethanol (12\% v/v) as their only fluid source for 5 months were then tested in the 8-arm radial arm maze discrimination task (Krazem et al., 2003). Adult mice began receiving this treatment in adolescence (2-3 months old) whereas aged mice began receiving this treatment at 16-18 months of age. After this time period all subjects took part in the discrimination task, which was then repeated 21 days later. The aged control mice displayed deficits in relational memory (but not procedural) and retention across the 21 day interval between the two testing sessions. These effects were reversed in aged rats that received ethanol. In contrast adult rats that received ethanol displayed a deficit in relational memory, but no deficit in retention. These results indicate that ethanol affect memory domains in very different ways at different stages of development. 
A similar effect was found in adult and aged C57B1/6 mice that received long-term ethanol exposure. Aged mice that received ethanol for 5 months performed significantly better in an 8-arm radial arm maze discrimination task than mice that started receiving the ethanol treatment in adolescence (Krazem, Marighetto, Higueret, and Jaffard, 2003). These findings suggest that long-term ethanol exposure is more damaging to developing adolescent brains, while potentially alleviating some cognitive deficits that arise with aging (Krazem, Marighetto, Higueret, and Jaffard, 2003). These two studies suggest that older brains can recover more easily from chronic ethanol exposure, but more research in this area is required before it can be determined what the true extent of the harmful nature of ethanol to the older brain is.

\section{The current study}

To determine whether ethanol administered at different stages of neurological development effects spatial and recognition or non-spatial memory differently, this study aims to compare the effect of ethanol on spatial and non-spatial memory, in rats aged approximately 8-12 weeks or 9-18 months. The NOR task trials will be used to test both nonspatial and spatial memory. It is predicted that saline subjects' performance may improve over time within and across days, while ethanol subjects will not due to ethanol-produced forgetting (Uzbay, and Kayaalp, 1995).

For cycle 1 of NOR testing it is predicted that as ITI time increases, a forgetting curve will present itself for ethanol-treated subjects in both non-spatial and spatial NOR task trials. Similarly, to subjects with hippocampal lesions that undergo NOR trial testing (Broadbent et al., 2010; Cohen and Stackman, 2015), no preference for either objects (equal approaches and/or time spent with both objects) or a preference for the familiar object that is no longer recognised from $\mathrm{T} 1$ could indicate hippocampal impairment from previous ethanol exposure. 
Due to the shorter ITIs implemented in this study, it is predicted that control subjects will still have intact memory processes (displayed as a preference for the novel object) even after the longest delay between phases, as seen in previous research with normal NOR task performance (Ennaceur and Delacour, 1988; Reger, Hovda, and Giza, 2009; Broadbent et al., 2010; Schenk et al., 2011). Performance is expected to be impaired for younger ethanol treated rats in comparison to older ethanol treated rats, due to heightened susceptibility of the adolescent brain (particularly the hippocampus) to the detrimental effects of ethanol (Swartzwelder, Wilson, and Tayyeb, 1995; Pyapali, Turner, Wilson, and Swartzwelder, 1999 Sircar and Sircar, 2005) and apparent lack of susceptibility of the older, previously unexposed brain/hippocampus (Krazem et al., 2003).

In addition, it is predicted that spatial memory deficits exhibited by younger ethanol treated subjects will be sustained one week later, whereas memory deficits in non-spatial trials will no longer be displayed due to recovery of recognition memory capabilities, as evidenced in previous research (Sircar and Sircar, 2005; Cippitelli et al., 2010). Older ethanol treated rats are predicted to display no remaining memory impairments for either type of trial.

\section{Method}

\section{Subjects}

The sample consisted of 76 Male Sprague-Dawley rats aged approximately 8-12 weeks $(n=56)$ and 9-18 months $(n=20)$. However an insufficient amount of the rats aged 918 months met the initial criteria of a minimum of 20 seconds exploration time in $\mathrm{T} 1$, so only younger rats were used for further analyses. Not all the younger rats met this criteria either resulting in varied numbers of ethanol and control rats for each trial type (3-9 ethanol rats, 10-16 controls). Additionally, several rats that met the initial criteria but either explored only one or none of the objects in $\mathrm{T} 2$ were eliminated from further analyses in order to gain a fair 
assessment of discriminatory capabilities. Assignment to groups (ethanol or control) was at random. The young rats weighed between $170-257 \mathrm{~g}$. Prior to testing, the rats were housed in groups of four in standard polycarbonate cages. These were located in a temperature $\left(21^{\circ} \mathrm{C}\right)$ and humidity (55\%) controlled colony which was maintained on a $12 \mathrm{~h}$ light/dark cycle with lights on at 07:00. The subjects were handled regularly, and had free access to food and water (outside of the testing phase time). All subjects were bred in the vivarium located at Victoria University of Wellington.

\section{Treatment}

The ethanol/vehicle was provided over a 5 day period consisting of one $1.0 \mathrm{~g} / \mathrm{kg}$ injection per day. This amount was chosen as it has been proven to be effective in producing memory impairments (Ryabinin, Miller, \& Durrant, 2002; Hayes, Deeny, Shaner, \& Nixon, 2013). Testing began 2 days following the last injection.

\section{Apparatus}

\section{Rota-rod.}

The first rota-rod model used consisted of a $70 \mathrm{~mm}$ diameter rotating plastic rod covered in sandpaper (to assist with grip). This was one lane in total semi-enclosed by white plastic walls and suspended above a plastic base.

The second rota-rod model used was a Panlab/Harvard Apparatus model (GmbH, Germany) with four lanes measuring $75 \mathrm{~mm}$ each in width. The rod itself was $60 \mathrm{~mm}$ in diameter. This apparatus was fully composed of plastic.

\section{Nor arena.}

The NOR arena is a $42 \mathrm{~cm} \times 42 \mathrm{~cm} \times 30 \mathrm{~cm}$ rectangle chamber with transparent Pyrex walls enclosed in a sound-proof cupboard (Medical Associates, ENV-001). A video camera 
was positioned above the chamber to record all activity. Two different sets of thirteen

different pairs of objects were used for cycle 1 and 2. See tables 1 and 2 below for objects

used

Table 1

Cycle 1 Novel Object Recognition (NOR) task items.

\begin{tabular}{ll}
\hline Object & Description \\
\hline Beer bottle & A brown glass bottle with long narrow neck \\
Can & A 355 ml metal can \\
Graduated cylinder & A short, clear plastic graduated cylinder \\
Shot glass & A short, transparent shot glass \\
Pink hole punch & A pink metal hole puncher, pressed down with squared edges \\
Beaker & A tall glass beaker \\
Glass & A short, purple round glass \\
Mug & A tall white ceramic mug with handle \\
Egg cup & A small white ceramic egg cup \\
Funnel & A white plastic funnel placed upside down during testing \\
Tuna can & A metal tuna can with no label \\
Plastic bottle & A clear plastic bottle 700ml \\
Decorative dish & A small black ceramic plate \\
\hline
\end{tabular}

Table 2

Cycle 2 Novel Object Recognition (NOR) task items.

\begin{tabular}{ll}
\hline Object & Description \\
\hline Tea-light holder & A small, round, purple shallow tea-light holder made of \\
& glass \\
Milk bottle & A tall, glass milk bottle \\
Palette & A small, plastic painting palette with six wells \\
Baking mould & A round, metal baking mould, placed upside down during \\
& testing \\
Round container & A small, round, plastic container with a white screw-on cap \\
Glass holder & A short, red, square glass holder \\
Teacup & A dark blue ceramic teacup with no handle \\
Salt shaker & A short glass salt shaker with a metal cap \\
Rice mould & A plastic, pink triangular rice mould \\
Glass bottle & A short, round glass container with an orange cap \\
Pencil sharpener & A small, pink, rectangular pencil sharpener \\
\hline
\end{tabular}




\section{Procedure}

On each day of the 5 ethanol/saline pre-treatment motor coordination was assessed using a rota-rod. Initially an older model of the rotor rod was used to assess motor coordination, with the rotor rod was set at 30 revolutions per minute (RPM). This was replaced by a newer model which was used for subsequent groups of rats, with the speed set at 20 RPM. Each rat was placed upon the rod 0, 20, and 40 minutes after saline or ethanol exposure and timed for endurance. If the rat remained on the rod for longer than 10 minutes the duration time was recorded as 600 seconds.

In both of the two days following the last injection subjects explored the NOR arena sans-objects for 5 minutes per day. After this the experimental trials commenced. For the procedure testing non-spatial memory, at time 1 the subject was placed in a chamber which included two identical objects. They were given 3 minutes to explore the objects. Following this, the subject was returned to the home cage while an inter-trial intervals (ITI) of 1, 3, or 5 minutes was implemented, in which time one object was replaced with a novel object (for non-spatial trials) or moved to a new location within the experimental chamber (for spatial trials) Subsequently the subject was placed back in the chamber for another 3 minutes. Video recordings of each trial were later viewed and amount of approaches and length of time of each approach was recorded.

\section{Exploratory behaviour}

Exploratory behaviour was defined when the rats' nose came within one centimetre of the object. This excluded rearing behaviour and/or climbing on top of the object. In order for the data from each NOR trial to be considered reliable, the rat was required to explore both objects in $\mathrm{T} 1$ for a minimum of 20 seconds, and to explore both objects for any given amount of time in T2. All tests were conducted during the light portion of the cycle (between 0700 
and 1900). All procedures were approved by the Animal Ethics committee at Victoria University of Wellington.

\section{Data analysis}

Data were analysed using SPSS statistics package (SPSS Inc.; version 19.0 for Windows 7). To examine the effects of treatment on rota-rod endurance lever responding a 3 3 (time) x 5 (day) x group (2) repeated measures analysis of variance (ANOVA) was used. Time and day were used as within-subjects factors, while treatment (ethanol or saline) was used as a between subject factor. Performance on the two rota-rod apparatuses used were analysed separately

To examine the effects of treatment on approaches to or time spent exploring each object in T1 and T2 of NOR task trials, a 2 (time 1 or 2) x 4 (object novelty- sample, duplicate, familiar or novel) x 3 (ITI length of 1,3 , or 5 minutes) x 2 (group) repeated measures ANOVA was used. Time, novelty and ITI were used as within-subjects factors, while treatment (ethanol or saline) was used as a between subject factor. Non-spatial and spatial data were separately analysed, as were data from cycle 1 and cycle 2 .

To examine the effects of treatment on the proportion of approaches or proportion of time spent exploring the duplicate object in T1 and the novel object in T2 of NOR task trials, a 2 (object novelty) x 3 (ITI) x 2 (group) repeated measures ANOVA was used. Novelty and ITI were used as within-subjects factors, while treatment (ethanol or saline) was used as a between subject factor. Once again non-spatial and spatial data were separately analysed, as were data from cycle 1 and cycle 2 .

\section{Results and discussion}

\section{Rota-rod performance}




\section{Initial rota-rod model.}

Figure 1 shows the amount of time spent on the rota-rod for ethanol and vehicletreated rats. Saline-treated animals remained on the rota-rod for a longer duration at all time points compared to ethanol treated animals. While there is slight improvement for the saline group on days 1 and 2, the existence of practice effects is unclear, and performance otherwise remained relatively stable across time for both groups.

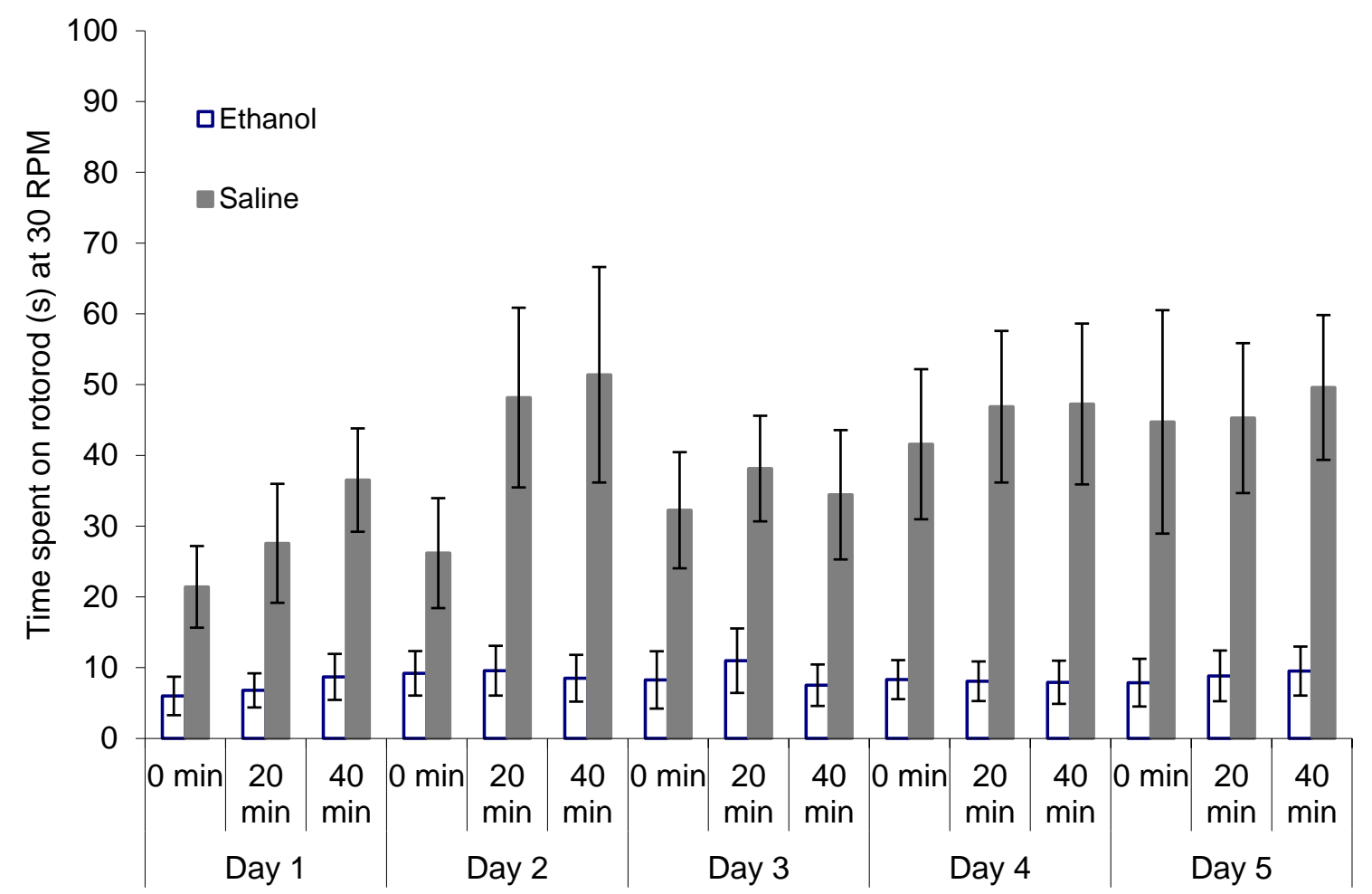

Figure 1 . Time spent on the old rota-rod following daily administration of saline $(0.9 \%$, IP) or Ethanol $(1 \mathrm{~g} / \mathrm{kg}, \mathrm{IP})$ at time zero, twenty, and forty. Error bars represent standard error of the mean.

As shown in table 1, a repeated measures ANOVA (time $\mathrm{x}$ day $\mathrm{x}$ group) revealed a significant main effect of day and time as shown in Table 1. This may indicate improvement over time, within and between sessions. A significant interaction between day and group, and time and group suggests that treatment altered performance. Post-hoc independent samples t- 
tests revealed that compared to ethanol treated subjects, control subjects remained on the rota-rod for a significantly longer duration at 0 minutes (control: $M=33.23, S D=33.83$; ethanol: $M=7.89, S D=2.21) t(126)=5.91, p<.001,20$ minutes $($ control: $M=41.20, S D=$ 33.28; ethanol: $M=8.85, S D=13.01) t(126)=7.58, p<.001$, and 40 minutes post- injection (control: $M=44.84, S D=35.74$; ethanol: $M=8.45, S D=12.06) t(126)=7.90, p<.001$.

Table 3. Analysis of variance following acute administration of saline or ethanol on initial rota-rod apparatus performance. ${ }^{*}$ Indicates significance at the .05 level.

\begin{tabular}{l|ll}
\hline Main effects & Day & $F(4,84)=4.497, p=.002^{*}$ \\
Time & $F(2,42)=5.392, p=.008^{*}$ \\
Day x time & $F(3.88,81.43)=1.040, p=.390$ \\
Day x group & $F(4,84)=3.197, p=.017^{*}$ \\
Time x group & $F(2,42)=3.919, p=.028^{*}$ \\
\end{tabular}

Figure 2 shows performance on the new rota-rod at 20 RPM at the 3 time points across the 5 days of testing. Saline treated animals remained on the rota-rod for a longer duration at all time points compared to ethanol treated animals, with performance across time being stable for the ethanol group, while the saline groups' performance varied within and between days. 


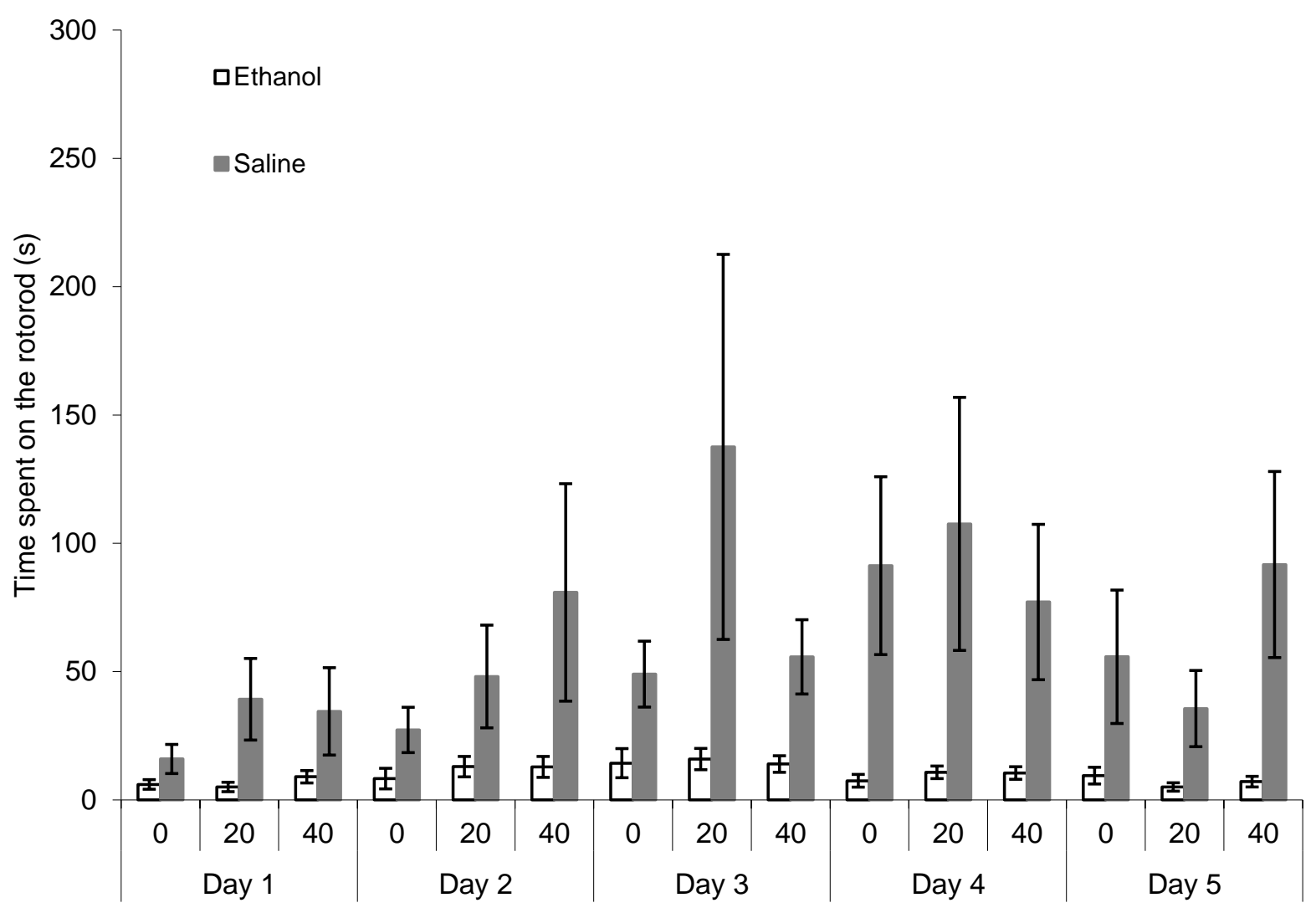

Figure 2. Time spent on the second rota-rod apparatus following daily administration of saline $(0.9 \%$, IP) or Ethanol $(1 \mathrm{~g} / \mathrm{kg}$, IP) at time zero, twenty, and forty. Error bars represent standard error of the mean.

Table 4 shows the results of a repeated measures ANOVA (time $\mathrm{x}$ day $\mathrm{x}$ group), which revealed a significant main effect of day and time. This may indicate improvement over time, within and between sessions. A significant interaction between day and group, time and group, and day time and group suggests that treatment altered performance. Posthoc independent samples t-tests revealed that compared to ethanol treated subjects, control subjects remained on the rota-rod for a significantly longer duration at 0 minutes (control: $M$ $=47.87, S D=49.99$; ethanol: $M=9.11, S D=13.21) t(87)=5.75, p<.001,20$ minutes (control: $M=73.60, S D=96.00$; ethanol: $M=10.03, S D=11.37) t(87)=5.25, p<.001$, and 
40 minutes post- injection (control: $M=144.99, S D=387.78$; ethanol: $M=10.77, S D=$ 10.55) $t(2.79)=7.90, p=.006$.

Table 4. Analysis of variance of rota-rod performance following acute administration of saline or ethanol. * Indicates significance at the .05 level, whereas ${ }^{\wedge}$ indicates a Greenhouse-Geisser correction of degrees of freedom following a violation of sphericity.

\begin{tabular}{|c|c|c|}
\hline \multirow[t]{6}{*}{ Main effects } & Day & $F(1.69,25.28)=3.54, p=.051^{\wedge}$ \\
\hline & Time & $F(1.29,19.35)=4.40, p=.041^{*}$ \\
\hline & Day $\mathrm{x}$ time & $F(2.04,30.61)=3.60, p=.039^{*}$ \\
\hline & Day x group & $F(4,60)=2.67, p=.041^{*}$ \\
\hline & Time $\mathrm{x}$ group & $F(2,30)=3.51, p=.043^{*}$ \\
\hline & Day $\mathrm{x}$ time $\mathrm{x}$ group & $F(8,120)=3.28, p=.002^{*}$ \\
\hline
\end{tabular}

\section{Cycle 1 novel object recognition testing}

Cycle 1 of NOR testing occurred 2 days after the final dose of saline or ethanol had been administered to each subject. Non-spatial and spatial trials were analysed separately, however for both trial types the dependent measures of approaches, proportion of approaches, exploration times, and proportion of exploration times were examined to determine whether one measure was more appropriate as a reflection of object preference than another.

\section{Non-spatial NOR trials}

\section{Approaches.}


$1 \mathrm{~A}$

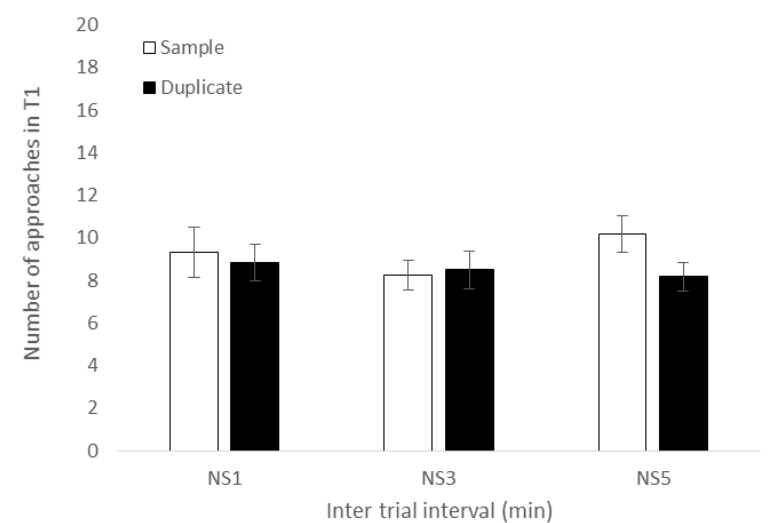

$2 \mathrm{~A}$

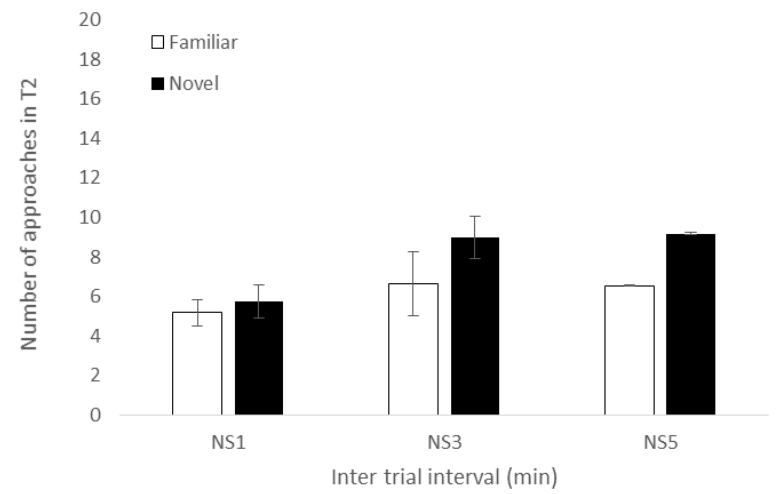

1B

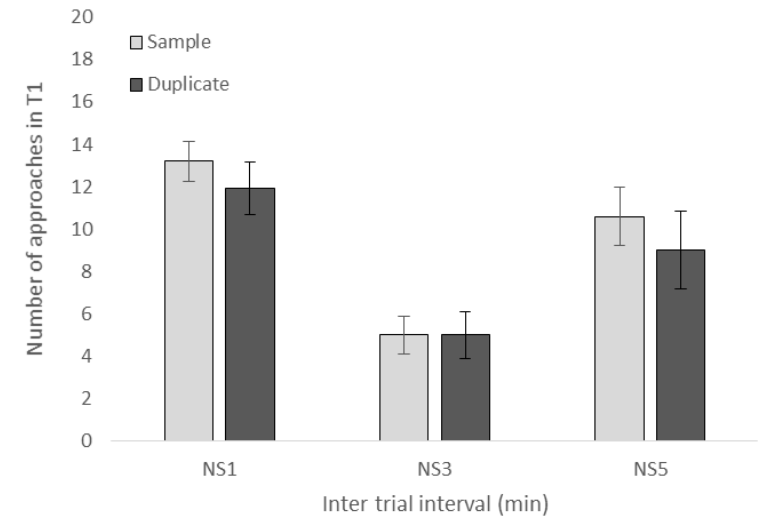

2B

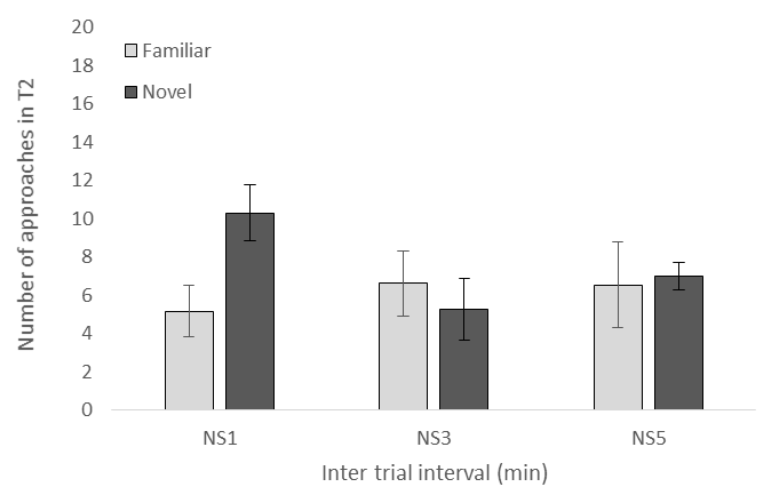

Figure 3. Number of approaches to each object in T1 (1) and T2 (2) for saline (A) and ethanol (B) treated animals across each cycle 1 non-spatial novel objection recognition trial. Error bars represent standard error means.

Figure 3 shows average number of approaches to the objects presented in time 1 and time 2 by both saline and ethanol-treated subjects. There was no obvious difference in the number of approaches to the sample and duplicate objects for saline-treated rats between trials (1A), however for ethanol treated rats there was an observable decrease in the amount of approaches made to both sample and duplicate objects between NS1 and NS3 trials (1B). It is also evident from figure 3 that approaches to the novel object increased when longer ITIs beyond 1 minute were implemented for saline-treated animals (1B), and decreased with increasing ITIs for ethanol-treated subjects (2B). 
A group $\mathrm{x}$ ITI $\mathrm{x}$ time $\mathrm{x}$ novelty repeated measures ANOVA was conducted to assess between and within-subject differences in amount of approaches to objects in both T1 and T2 across all 3 ITIs for non-spatial trials. As depicted in table 3, a significant effect of ITI was found, $F(1,44)=3.45, p=.041$, as well as a significant effect of time, $F(1,44)=8.28, p=$ .006. A significant interaction between time and ITI was also revealed, with post-hoc comparisons indicating that more approaches were made in total in T1 $(p=.006)$ of NS1 trials $(p=.041)$ compared to T1 and/or T2 of all other non-spatial trials. An additional significant interaction between group and ITI, $F(2,44)=7.03, p=.002$, indicates that this increase in approaches in T1 of NS1 trials is due to ethanol-treated subjects, as presented in figure 3.

A

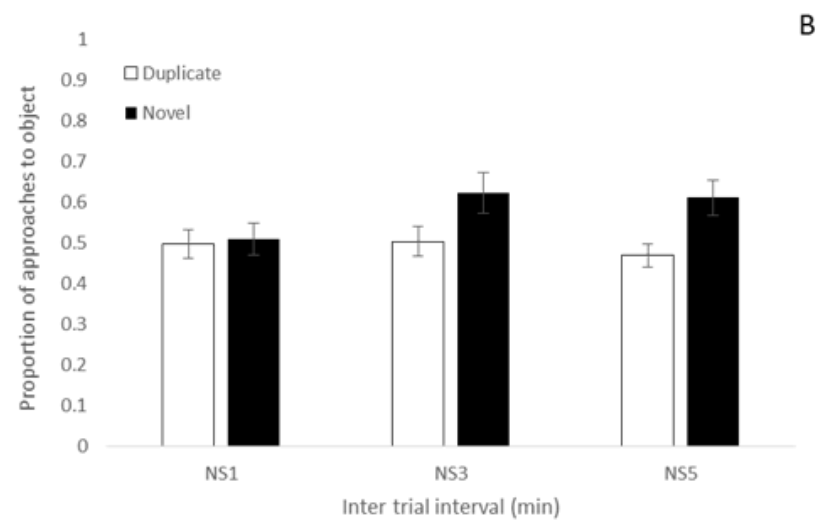

$B$

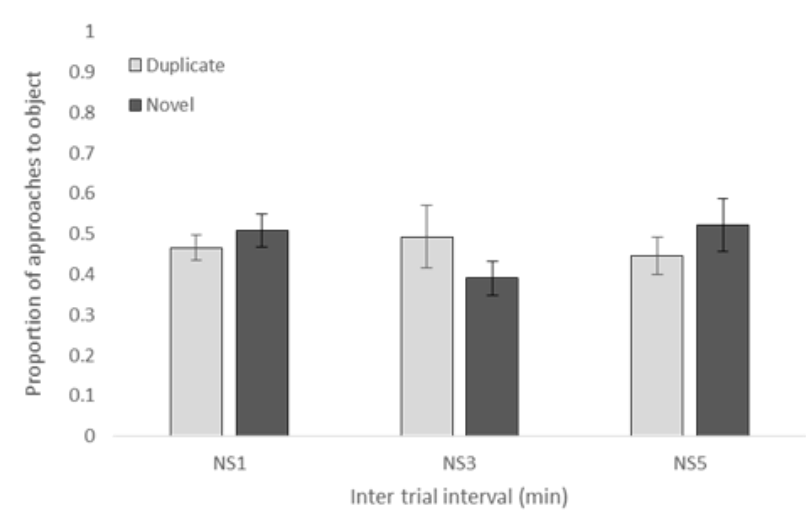

Figure 4. Proportion of number of approaches to duplicate objects in T1 and replacement novel objects in T2 for saline (A) and ethanol (B) treated animals across each cycle 1 nonspatial novel objection recognition trial. Error bars represent standard error means.

Figure 4 shows the average proportion of approaches made by both saline and ethanol treated subjects towards the duplicate object in $\mathrm{T} 1$ and the novel object which replaced it in T2. This allows for a direct comparison of approach data between T1 and T2. Saline-treated subjects allocated close to 0.5 of their approaches to the duplicate object in T1 of all nonspatial trials, while making a proportion of approaches slightly higher than chance to the novel object in T2 of NS3 and NS5 trials (A). There was minimal difference in the proportion 
of approaches made by ethanol treated subjects to either the duplicate or novel objects for any trial, with the exception of NS3 trials where the proportion of approaches made to the novel object in $\mathrm{T} 2$ was less than 0.5 . From this figure there was is no apparent position bias from subjects affecting where approaches were made, nor an effect of ITI on the proportion of approaches made to the novel object for either group.

A further group $\mathrm{x}$ novelty $\mathrm{x}$ ITI repeated measures ANOVA was conducted to assess differences in proportion of approaches to the duplicate object in $\mathrm{T} 1$ and the novel object in $\mathrm{T} 2$ of all non-spatial trials. Results revealed an effect of novelty $F(1,44)=4.46, p=.040$ with a higher proportion of approaches made towards the novel object compared to the duplicate $(p=.040)$ and an effect of group $F(1,44)=4.62, p=.037$, with the saline group having a higher proportion of approaches towards the novel object compared to the ethanol $\operatorname{group}(p=.037)$.

\section{Exploration time.}



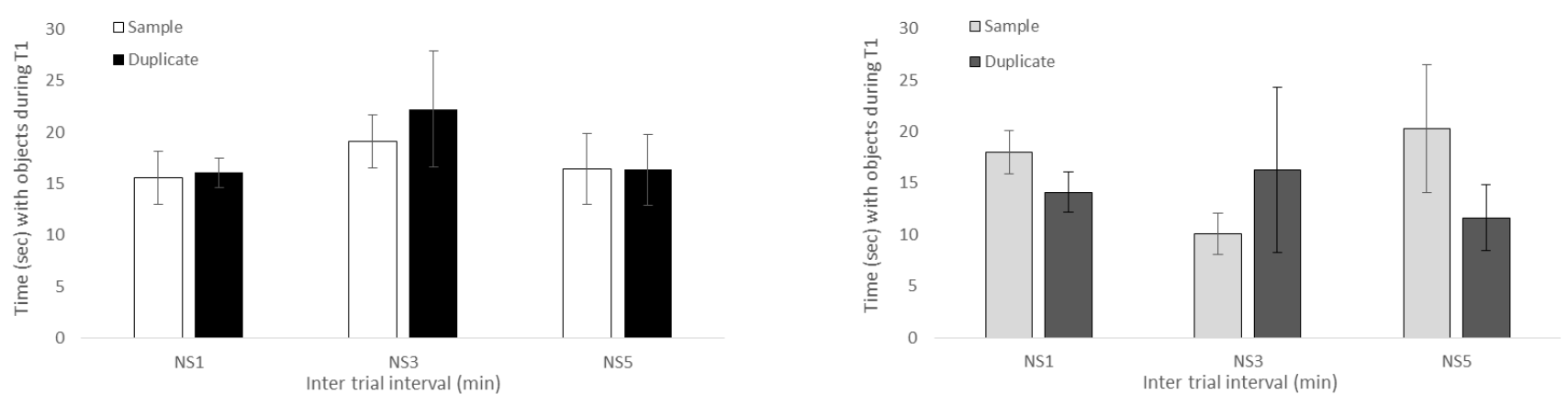

$2 \mathrm{~A}$
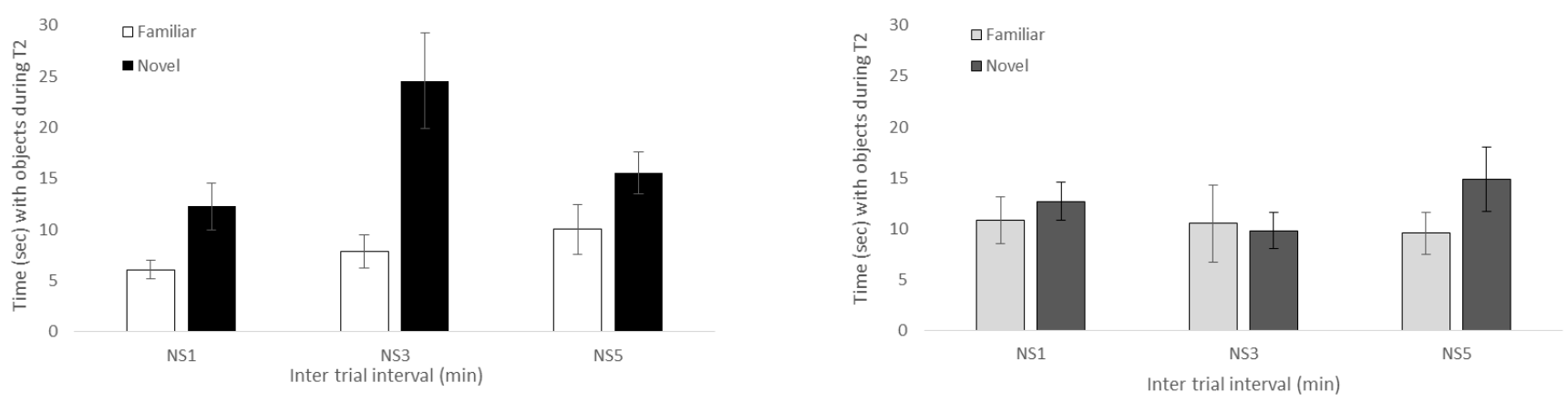

Figure 5. Time spent exploring both objects in T1 (1) and T2 (2) for saline (A) and ethanol (B) treated animals during the cycle 1 non-spatial objection recognition task. Error bars represent standard error means.

Figure 5 shows average time spent exploring each object presented in time 1 and time 2 by both saline and ethanol-treated subjects. Total exploration time was no more than 45 seconds for either T1 or T2, however ethanol treated subjects spent less time exploring objects compared to saline treated subjects at both times. For saline-treated subjects there was a slight difference in time spent exploring sample and duplicate objects for NS3 trials in T1 (1A), whereas time spent exploring both objects in T1 appeared relatively equal for NS1 and NS5 trials. At T2 saline-treated subjects spent more time exploring the novel object in comparison to the familiar object, which was most prominent in NS3 trials. This pattern suggests that saline treated subjects were displaying a novelty preference across all ITIs. For ethanol-treated subjects there was a visible difference in exploration time of each object at T1 (1B), and T2 (2B), but in no discernible pattern. It is once again difficult to ascertain whether 
this is a result of forgetting or simple lack of motivation to explore, as suggested by lower exploration times in general.

A repeated measures ANOVA revealed a significant effect of novelty, $F(1,44)=$ $5.32, p=.026$, which post-hoc comparisons indicated to be due to an increased amount of time spent exploring the duplicate object in $\mathrm{T} 1$ and the replacement novel object in $\mathrm{T} 2 \mathrm{in}$ comparison to the sample object at both $\mathrm{T} 1$ and $\mathrm{T} 2$. A significant effect was also found for time $F(1,44)=15.98, p<.001$ due to an increased amount of total time spent exploring objects in T1 compared to T2 $(p<.001)$. A significant interaction between novelty and group was revealed $F(1,44)=5.28, p=.026$, in addition to a significant interaction between novelty and time, $F(1,44)=7.83, p=.008$.

A

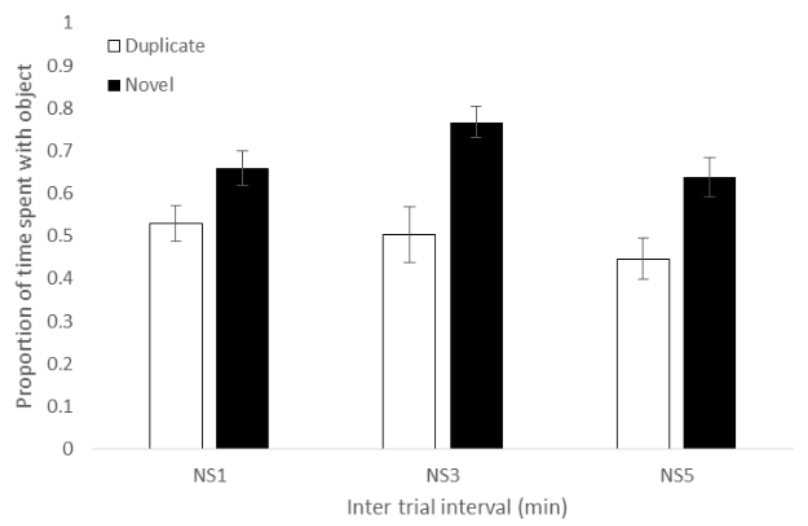

B

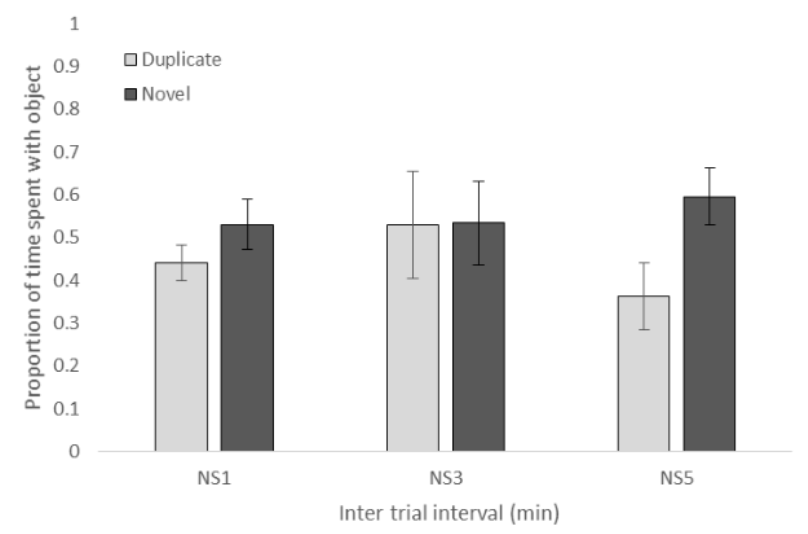

Figure 6. Proportion of time spent exploring duplicate objects in T1 and replacement novel objects in $\mathrm{T} 2$ for saline (A) and ethanol (B) treated animals during the cycle 1 non-spatial novel objection recognition task. Error bars represent standard error means.

Figure 6 shows the average proportion of time spent exploring the duplicate object in $\mathrm{T} 1$ and the novel object which replaced it in T2 for all non-spatial NOR trials for both saline (A) and ethanol-treated subjects (B) for the duplicate object in T1 and the novel object which replaced it in T2. Saline-treated subjects allocated almost equal time with each object in T1 of each trial (A), whereas the proportion of time spent with the duplicate object in T1 varied 
across all 3 trials for ethanol-treated subjects (B). Saline-treated subjects consistently spent a larger proportion of time with the novel object across all trials in T2, while ethanol-treated subjects spent only slightly over 0.5 of their total time with the novel object. From this figure there it can be established that there was no apparent position bias from subjects affecting exploration, nor an effect of increasing ITI on the proportion of time spent exploring to the novel object for either group.

A further repeated measures ANOVA was conducted to assess differences in proportion of time spent exploring the duplicate object in $\mathrm{T} 1$ and the novel object in $\mathrm{T} 2$ for all non-spatial trials. A significant effect of novelty was revealed $F(1,44)=20.11, p<.001$, with a higher proportion of time spent exploring the novel object compared to the duplicate for both groups, and an effect of group $F(1,44)=6.81, p=.037$, with the saline group spending a greater proportion of time exploring the duplicate and novel objects compared to the ethanol group.

It is shown from these results that when performance in the non-spatial NOR task was measured by total approaches towards objects, no group differences in approaches to each object were found after any ITI. However, when performance was measured by proportion of approaches, results showed that saline treated subjects allocated a significantly larger proportion of approaches to the novel object in T2 of cycle 1 non-spatial trials compared to ethanol treated subjects.

When performance in the non-spatial NOR task was measured by total time and proportion of time spent exploring each object, saline treated subjects explored objects more in general compared to ethanol treated subjects. However, both groups spent a larger proportion of time exploring the novel object in $\mathrm{T} 2$ in comparison to the duplicate in $\mathrm{T} 1$ which indicates some preference for the novel object. 
As there was no overall effect of ITI, it is difficult to determine whether these differences are due to impaired recognition memory across all trials on part of the ethanol group or a lack of motivation to explore objects altogether, thus it cannot be concluded whether these results support the hypotheses.

\section{Spatial NOR trials.}

\section{Approaches.}

$1 \mathrm{~A}$

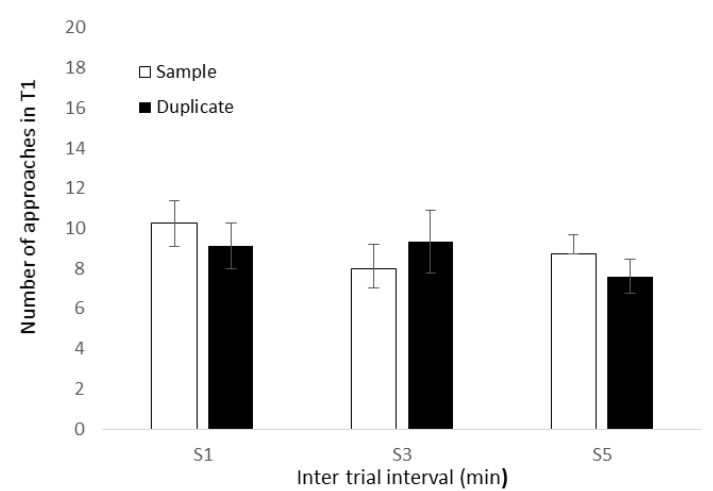

$2 \mathrm{~A}$

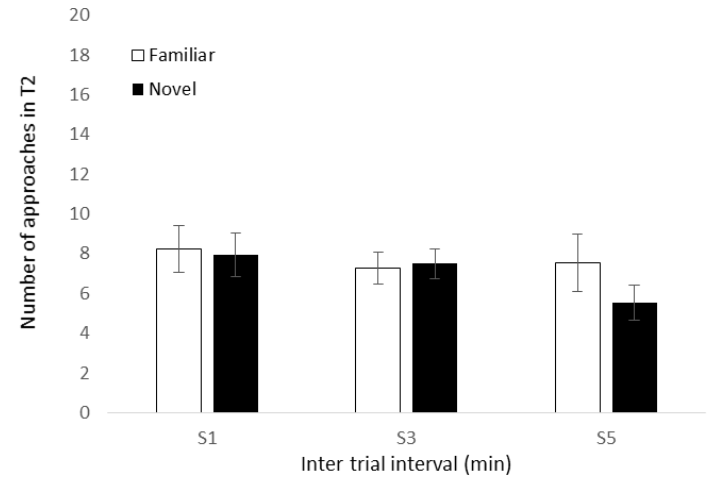

$1 \mathrm{~B}$

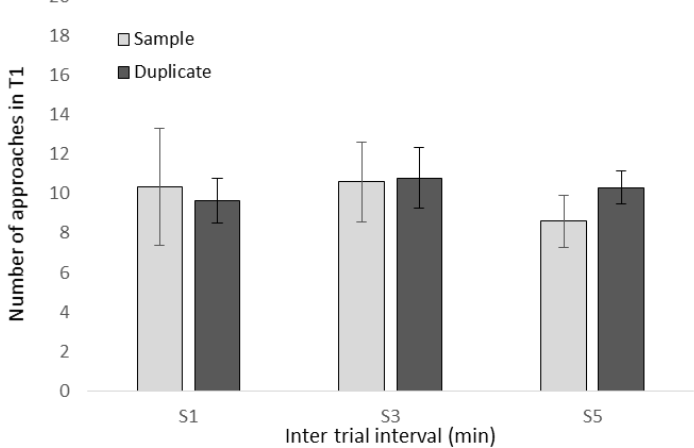

$2 \mathrm{~B}$

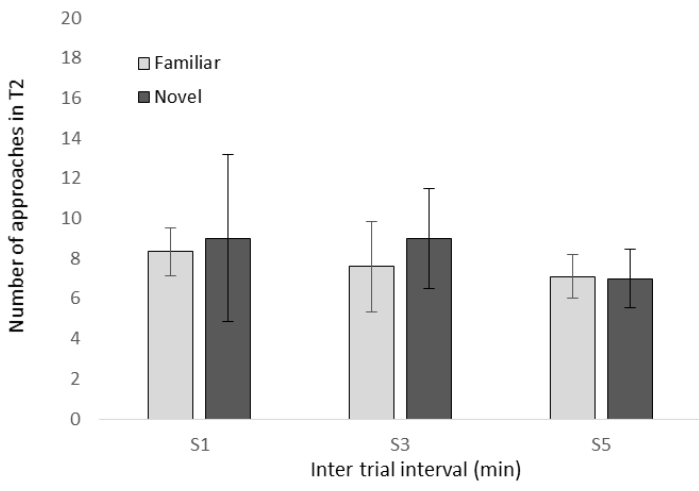

Figure 7. Number of approaches to each object in T1 (1) and T2 (2) for saline (A) and ethanol (B) treated animals across each cycle 1 spatial novel objection recognition trial. Error bars represent standard error means.

Figure 7 shows the amount of approaches made by saline (A) and ethanol-treated subjects (B) to the sample and duplicate objects in T1 (1) and the familiar and novel objects in T2 (2) of spatial NOR task trials. For saline-treated subjects there were minimal differences in the amount of approaches made to each object in both T1 and T2, with the exception of S5 trials where there was a more pronounced difference between approaches 
made to the familiar and novel objects in T2. Ethanol treated subjects had slight differences between approaches to each object in both $\mathrm{T} 1$ and $\mathrm{T} 2$, although interestingly made slightly more approaches to the novel object over the familiar object in T2 of both S1 and S3 trials, a trend not seen in the saline treated subjects.

A repeated measures ANOVA was conducted to assess between and within-subject differences in amount of approaches to objects in both T1 and T2 across all 3 spatial NOR task trials. A significant effect of time was revealed $F(1,55)=7.99, p=.007$, with pairwise comparisons indicating that more approaches were made to both objects in $\mathrm{T} 1$ compared to $\mathrm{T} 2$.

A

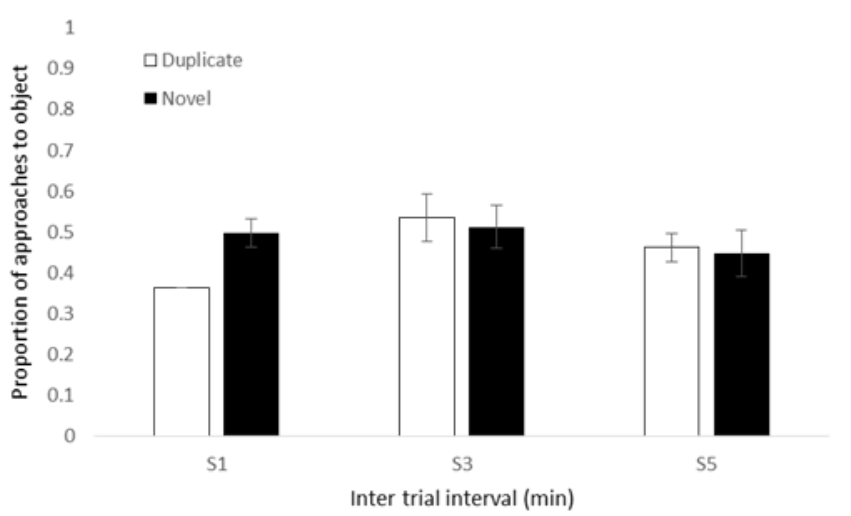

B

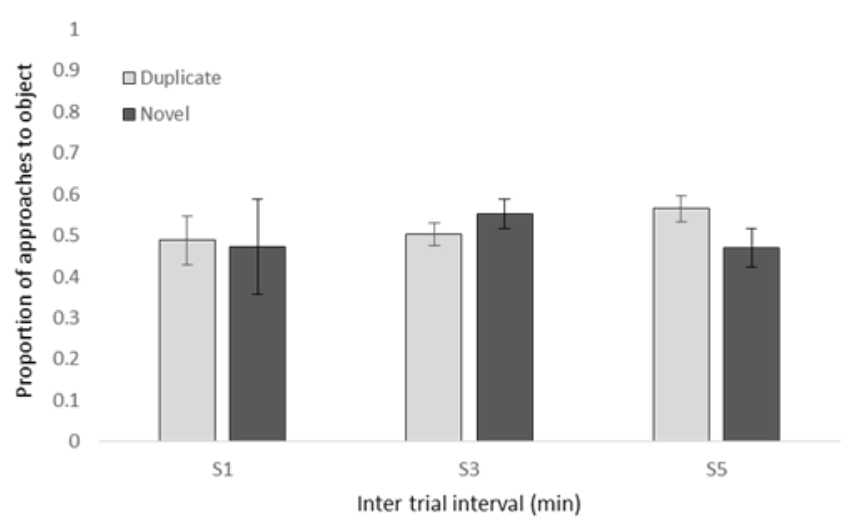

Figure 8. Proportion of number of approaches to duplicate objects in T1 and replacement novel objects in T2 for saline (A) and ethanol (B) treated animals across each cycle 1 spatial novel objection recognition trial. Error bars represent standard error means.

Figure 8 shows the average proportion of time spent exploring the duplicate object in T1 and the novel object which replaced it in T2 for all spatial NOR trials. Saline-treated subjects allocated approximately equal time with each object in T1 of S3 and 5 trials, however only $40 \%$ of approaches were made towards the duplicate object in S1 trials. Ethanol-treated subjects were close to $50 \%$ in the proportion of approaches they made to 
objects in both T1 and T2 of spatial NOR trials. No evidence of location bias or a forgetting curve in T2 due to increasing ITIs is evident.

A further repeated measures ANOVA was conducted to assess differences in proportion of approaches to the duplicate object in $\mathrm{T} 1$ and the novel object in $\mathrm{T} 2$ for all spatial trials. No significant effects or any interactions between effects were found.

\section{Exploration times.}

$1 \mathrm{~A}$

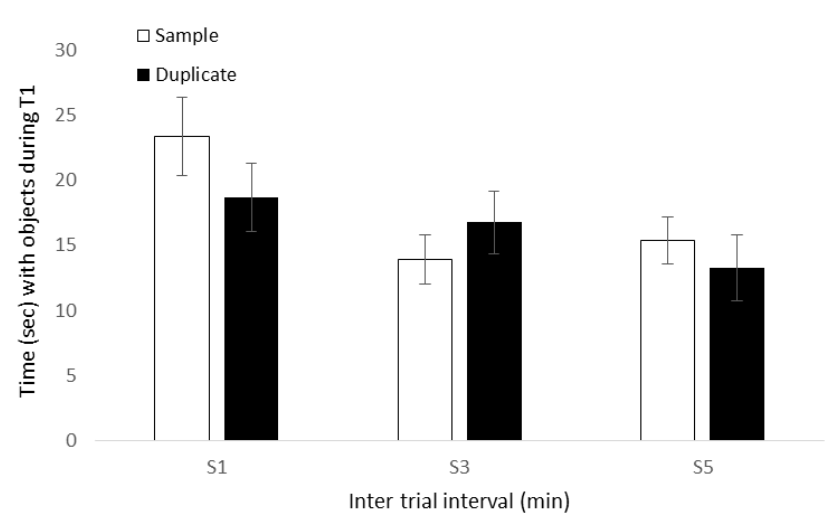

$2 \mathrm{~A}$

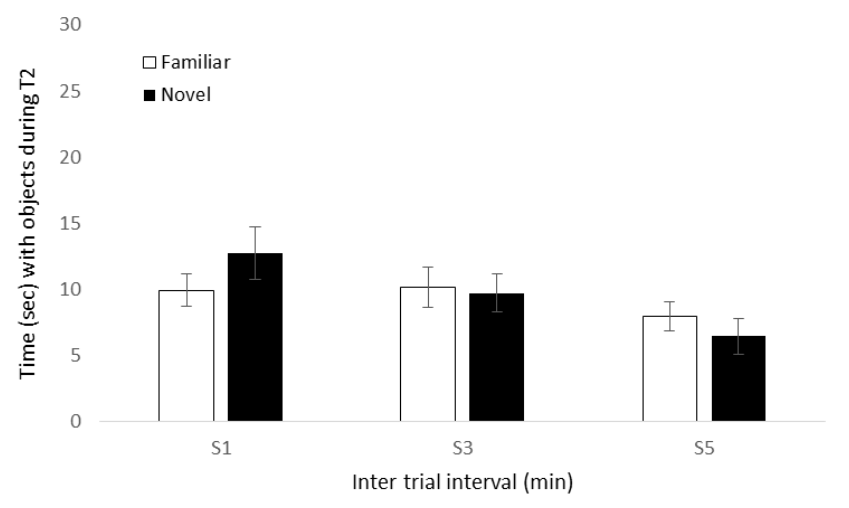

$1 B$

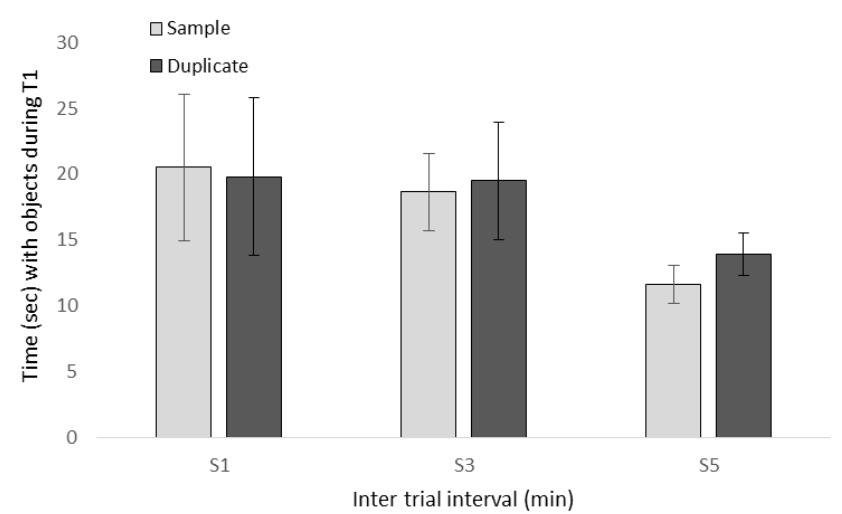

$2 \mathrm{~B}$

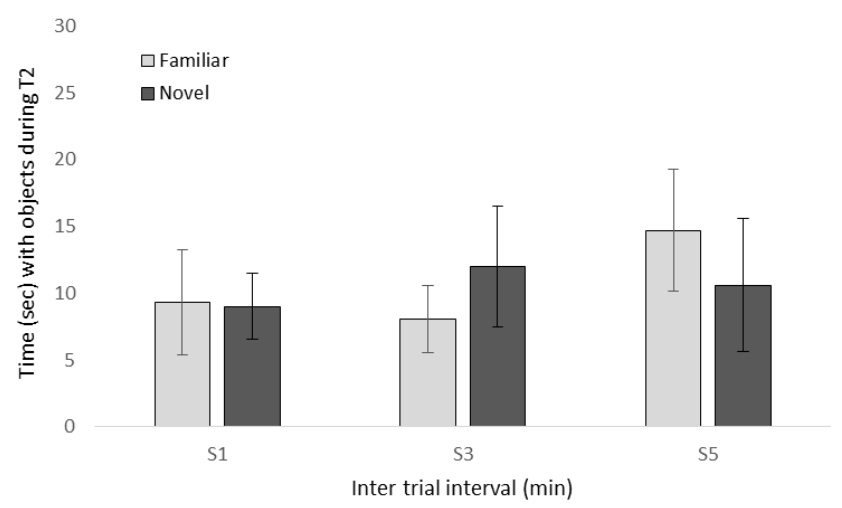

Figure 9. Time spent exploring both objects in T1 (1) and T2 (2) for saline (A) and ethanol

(B) treated animals during each trial of the cycle 1 spatial novel objection recognition task.

Error bars represent standard error means.

Figure 9 shows average time spent exploring each object presented in time 1 and time 2 by both saline and ethanol-treated subjects. For saline-treated subjects the most noticeable difference in $\mathrm{T} 1$ is between times spent exploring the sample and duplicate objects in $\mathrm{S} 1$ 
trials. Ethanol-treated subjects spent relatively equal time exploring both objects in $\mathrm{T} 1$ across all 3 trials, although less time was spent exploring objects overall in S5 trials. Both groups had lower overall exploration times in T2 compared to T1. Saline-treated subjects had a noticeable increase in time spent exploring the novel object in S1 trials, an effect which reversed with increasing ITIs. Ethanol-treated subjects had an increase in time spent exploring the novel object in comparison to the familiar object in S3 trials, and a contrasting decrease in S5 trials, indicating a possible forgetting effect due to a longer ITI.

A repeated measures ANOVA assessing differences in time spent exploring objects for spatial NOR trials revealed a main effect of time $F(1,55)=29.83, p<.001$. Pairwise comparisons indicated that subjects spent significantly more time exploring objects in $\mathrm{T} 1$ in comparison to T2 $(p<.001)$.

A

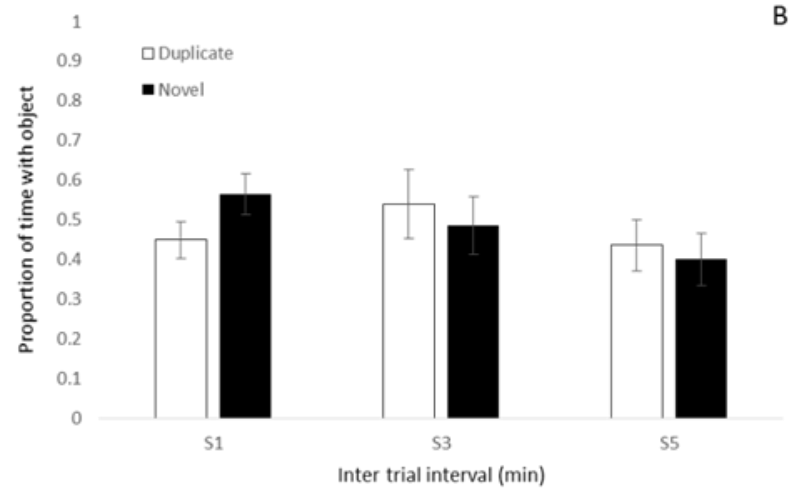

B

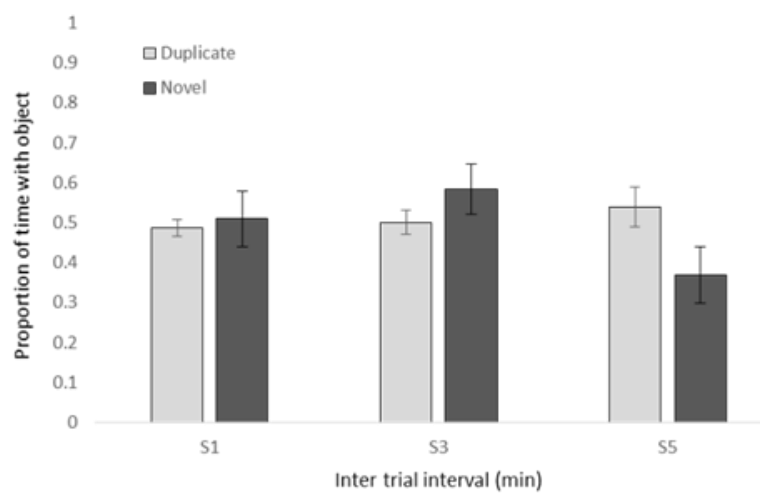

Figure 10. Proportion of time spent exploring duplicate objects in $\mathrm{T} 1$ and replacement novel objects in $\mathrm{T} 2$ for saline (A) and ethanol (B) treated animals during the cycle 1 spatial novel objection recognition task. Error bars represent standard error means.

Figure 10 shows the average proportion of time spent exploring the duplicate object in $\mathrm{T} 1$ and the novel object which replaced it in T2 for all spatial NOR trials for both saline (A) and ethanol-treated subjects (B) for the duplicate object in T1 and the novel object which replaced it in T2. Both groups allocated a relatively equal proportion of time to exploration of 
both objects in T1 and T2 with the except of T2 of S5 trials for ethanol subjects, where they spent less than $40 \%$ of their time with the novel object, again indicating a potential forgetting effect due to the implementation of a longer ITI.

A repeated measures ANOVA assessing difference in proportion of time spent exploring the duplicate object in $\mathrm{T} 1$ and the novel object in $\mathrm{T} 2$ revealed no significant main effects, or any interaction between effects.

These findings demonstrate that any measure of performance in cycle 1 of the spatial NOR task resulted in no significant between or within group differences in object preference after any ITI. This indicates that 5 consecutive days of ethanol administration followed by a 2 day recovery period did not lead to an impairment in memory in a capacity that would alter spatial NOR task performance.

\section{Cycle 2 novel object recognition testing}

Cycle 2 of NOR testing occurred 9 days after the final dose of saline or ethanol had been administered to each subject, and 7 days after cycle 1 of NOR testing had been completed. Once again non-spatial and spatial trials were analysed separately, with the dependent measures of approaches, proportion of approaches, exploration times, and proportion of exploration times all being examined to determine whether one measure is more appropriate as a reflection of object preference than another.

\section{Non-spatial NOR trials.}

Table 5. Significant effects obtained from analysis of various measures of performance in Cycle 2 non-spatial NOR trials. 


\begin{tabular}{r|ll} 
Approaches & Group & $F(1,25)=6.74, p=.016$ \\
Time & $F(1,25)=6.27, p=.016$ \\
Exploration time & Time & $F(1,25)=16.71, p<.001$ \\
Novelty x time & $F(1,25)=15.92, p=.001$ \\
Proportion of exploration time & Novelty & $F(1,25)=20.03, p<.001$
\end{tabular}

Table 5 displays all significant effects found when cycle 2 non-spatial NOR trial performance across all three ITIs was measured by approaches to each object, proportion of approaches, time spent exploring each object, and proportion of exploration time.

For approaches, a repeated measures ANOVA revealed a significant effect of group, with pairwise comparisons indicating that more approaches were made as a whole by the ethanol group, as well as a significant effect of time, due to more approaches as a whole being made in $\mathrm{T} 1$.

When performance was analysed by the proportion of approaches to the duplicate object in $\mathrm{T} 1$ and the novel object in $\mathrm{T} 2$, a repeated measures ANOVA revealed no significant main effects, or any interaction between effects.

For exploration time, a repeated measures ANOVA revealed a significant effect of time which pairwise comparisons indicated to be due to an increased amount of time spent exploring both of the objects in T1 compared to T2. A significant interaction was also found between novelty and time due to more time being spent with the sample object in T1 vs. T2 for both groups. 
A further repeated measures ANOVA was conducted to assess differences in proportion of time spent exploring the duplicate object in $\mathrm{T} 1$ and the novel object in $\mathrm{T} 2$ for all non-spatial trials. A significant effect of novelty was revealed with a higher proportion of time spent exploring the novel object compared to the duplicate for both groups.

These results show that when performance in cycle 2 of the non-spatial NOR task was measured by approaches, proportion of approaches, total time and proportion of time spent exploring objects, no between or within group differences were found after the implementation of any ITI. Although these similarities in performance between groups could reflect our prediction that the non-spatial memory of ethanol treated subjects would recover by the time of cycle 2 testing, it cannot be determined whether these results support our hypothesis as there is no indication that non-spatial memory was ever impaired in cycle 1 testing in the first place.

\section{Spatial NOR trials.}

Table 6. Significant effects obtained from analysis of various measures of performance in Cycle 2 spatial NOR trials.

\begin{tabular}{r|ll} 
Approaches & Time & $F(1,38)=17.03, p<.001$ \\
Exploration time & Time & $F(1,38)=15.91, p<.001$
\end{tabular}

Table 6 displays all significant effects found when cycle 2 spatial NOR trial performance was measured by approaches to each object, proportion of approaches, time spent exploring each object, and proportion of exploration time. 
A repeated measures ANOVA assessing between and within-subject differences in amount of approaches to objects in both T1 and T2 across all 3 spatial NOR task trials revealed a significant effect of time, with pairwise comparisons indicating that more approaches were made to both objects in T1 compared to T2. The same effect was found when exploration time was analysed, with pairwise comparisons indicating that subjects spent significantly more time exploring objects in $\mathrm{T} 1$ in comparison to T2.

Repeated measures ANOVAs assessing differences in both the proportion of approaches to the duplicate object in $\mathrm{T} 1$ and the novel object in $\mathrm{T} 2$ and proportion of exploration time revealed no significant main effects, or any interaction between effects.

From these results it is shown that any measure of performance in cycle 2 of the spatial NOR task revealed no significant group differences in approaches to each object were found after any ITI. This indicates that contrary to our hypothesis, treatment did not affect memory in a prolonged capacity that would alter spatial NOR task performance 9 days after administration of ethanol had ceased.

\section{General discussion}

This study aimed to investigate the effect of acute ethanol exposure on spatial and non-spatial memory at two stages of development. However due to a lack of subjects meeting the initial experimental criteria from the older ethanol treated group this became a study of the effect of a "binge-like" period of ethanol exposure on spatial and non-spatial memory in young rats aged 2-3 months only. We firstly hypothesised that ethanol treated subjects would remain on the rota-rod for a shorter period of time at all 3 time points across the 5 days of treatment compared to saline treated control subjects. Our hypothesis was supported when subjects were placed on rota-rods running at both 20 and 30 RPM, indicating that the ethanol treatment was effective to the point of producing motor co-ordination impairments. 
The effects of this pre-treatment on NOR performance was measured at two time periods. The first assessment took place 2 days following treatment in order to determine whether there was an effect on spatial and/or non-spatial memory. The second test occurred one week later in order to assess whether the hypothesised initial effects would dissipate over time.

NOR testing was conducted at various time intervals (ITIs) in order to generate a "forgetting curve". It was expected that preference for the novel object would decrease as ITI increased, as has been observed in other studies (Ennaceur and Delacour, 1988; Reger, Hovda, and Giza, 2009; Broadbent et al., 2010; Schenk et al., 2011). We also expected that the ethanol exposed rats would demonstrate compromised performance. For cycle 2 trials we hypothesised that memory deficits predicted to be exhibited by the young ethanol-treated subjects in cycle 1 spatial NOR trials would also be present one week later in cycle 2 of the testing phase, whereas memory deficits in non-spatial trials would no longer be displayed, with normal preference for the novel object being demonstrated. This was expected due to findings from previous literature where non-spatial memory was demonstrated to recover at a faster rate than spatial memory (Sircar and Sircar, 2005; Cippitelli et al., 2010). From the results we can conclude that all of our hypotheses for performance in the NOR task across both cycles were not supported.

When performance in cycle 1 non-spatial trials was measured by the proportion of approaches to the novel object in T2, saline treated subjects had a significantly larger proportion of approaches to the novel object compared to ethanol treated subjects. However, when this was measured as a proportion of time spent exploring the novel object, there were no differences between groups, with both saline and ethanol subjects showing a preference for the novel object across all ITIs that were implemented. 
These results contradict the findings of Cippitelli et al. (2010), where ethanol treated subjects displayed an impairment in novel object recognition and the ability to detect spatial change in an object exploration task when delays of only four minutes were imposed, something which occurred five days after the ethanol treatment had desisted. This pattern was found when performance was measured by both approaches and exploration time, suggesting that there is some overlap between the two. These results also contrast with effects of $2 \mathrm{~g} / \mathrm{kg}$ of ethanol once a week for 10 weeks. These rats displayed significantly worse performance in a spatial version of the DNMS task compared to controls at delays as small as 1 minute (García-Moreno and Cimadevilla, 2012). This was measured by both exploration times and proportion of time spent exploring the novel object/ novel placement.

Though other research has found that rodents that received acute exposure to ethanol display intact object recognition memory after short ITIs ( $<15$ minutes) (García-Moreno et al., 2002; Matthews et al., 2002; Berry and Matthews, 2004; García-Moreno and Cimadevilla, 2012), whether the current findings support the previous literature is dubious as novel object preference was only found for both groups in cycle 1 of non-spatial trials when measured as the proportion of time spent exploring the novel object. As this aforementioned previous research typically used multiple measures of performance for the same task, it is likely that the difference in the current study between measures of cycle 1 non-spatial NOR task novelty preference is a spurious result.

This lack of support is only further increased when the cycle 2 non-spatial results are considered, as neither group displayed a novel object preference after any ITI is imposed, nor a forgetting effect as ITI increased. This also contradicts Cippitelli et al. (2010), as ethanol treated subjects still displayed impairments in spatial memory in the object exploration task 10 weeks after treatment had desisted, showing an ability to detect spatial change after delays of four minutes. Results found by Matthews et al. (2002) with adult rats, and Berry and 
Matthews (2004) with adolescent mice also showed a clear disparity between non-spatial and spatial MWMT performance after acute administration of ethanol, although only in doses of $1.5 \mathrm{~g} / \mathrm{kg}$ and above.

While ethanol treated subjects failed to display a forgetting curve across increasing ITIs despite previous literature suggesting an effect would occur, it is even more curious that saline treated controls subjects did not display consistent novelty preference in the testing phase of any NOR task trial. Novelty preference in normal subjects has been found consistently across a wide range of both non-spatial (Ennaceur and Delacour, 1988; Reger et al., 2008; Hovda, and Giza, 2009; Antunes and Biala, 2012) and spatial studies (Ennaceur and Meliani, 1992; Ennaceur, Neave, and Aggleton, 1997; Weible, Rowland, Pang, and Kentros, 2009) involving the NOR paradigm. This makes it difficult to interpret the results of the ethanol-treated group in comparison, and could mean that one or more extraneous variables are influencing the current results. One of these potential extraneous variables could be the handling of the subjects that occurred between T1 and T2, something which occurred frequently in a short period of time due to ITI lengths, and may have distressed the animals.

The fact that no forgetting curve was found for ethanol treated subjects despite a wide breadth of literature demonstrating the detrimental effect of ethanol on spatial memory and even non-spatial memory could be due to a number of factors. In this study we narrowed down the delay in which a forgetting curve could occur to a window of between 1 and 5 minutes. As multiple studies have found memory deficits when longer delays were implemented, perhaps a forgetting effect would have appeared in the current study had this also been the case. García-Moreno and Cimadevilla (2012) found that both control and alcohol-treated rats that received both acute and chronic treatment performed best in memory tasks with a 15 minute delay, in comparison to delays of 1 minute or 1 hour. However, Schenk et al. (2011) found that control rats that self-administered saline prior to undergoing 
the NOR task displayed a forgetting curve as a function of ITIs 1-15 minutes long, something which was only revealed when performance was measured by the proportion of approaches to the novel object. This discrepancy could be due to differing habituation times between studies, or the amount of handling subjects received between the familiarisation and testing phases.

A further possible explanation is a general decrease in movement or hypolocomotion as a result of ethanol withdrawal. Logan, Seggio, Robinson, Richard, and Rosenwasser (2010) found this effect when they administered C57BL/6J mice a short-term 4 day CIE treatment (16 hours of ethanol vapour alternating with 8 hour withdrawal periods), which resulted in a temporary albeit significant reduction in both running-wheel activity and freerunning circadian rhythms compared to controls, lasting approximately one week after the final ethanol vapour exposure. This could potentially explain the lower amounts of ethanol subjects meeting the minimum exploration criteria in $\mathrm{T} 1$ in both cycle 1 and 2 trials, as cycle 1 trials commenced two days after the final dose of ethanol was administered, while cycle 2 trials occurred only slightly over one week following this.

\section{Limitations}

Sample sizes in the current study were substantially decreased from initial amounts due to lack of exploration in T1, particularly ethanol subjects where the proportion of rats that met the criteria was very small. It known that intraperitoneal ethanol injections can result in inflammation to the administration site for up to 3 weeks (In, Kim, Park, and Kim, 2014), which may result in a restriction of movement due to associated pain. Considering that the methodology of the current study involved administering 5 consecutive daily injections, the effect may have been aggravated even more so, even when the side of injection site was alternated each day. 
As the cycle one NOR testing took place two days after the last dose of ethanol, withdrawal may have also played a part in the performance of the ethanol-treated rats during the trials. In the chronic intermittent ethanol (CIE) model of ethanol exposure repeated withdrawal is a key component, and during this period rats often display increased susceptibility to seizures, anxiety, and behavioural excitability (Cagetti et al., 2003). Cagetti et al. (2003) observed a significant increase in anxiety in CIE exposed rats compared to control subjects in the elevated plus maze, an effect that lasted for at least two days after ethanol cessation.

\section{Future directions}

Future investigation in this area could potentially solve the issue of lack of sufficient T1 exploration times by modifying the length of time for the T1 period to allow more opportunity for exploration. When Ennaceur and Delacour (1988) found that their initial NOR experiment was not sensitive enough to retention duration, they modified the test so that instead of $\mathrm{T} 1$ being a fixed amount of time, the phase was only over when the minimum 20 second exploration criteria were met. If this modification was used in the future with the NOR paradigm it could allow for increased familiarisation of objects, and more subjects could potentially be included in further analyses, yielding a different pattern of results.

The effect of multiple schedules of administration (acute exposure, chronic exposure, chronic intermittent exposure) on NOR task performance could also be assessed. This would allow researchers to determine if one model of binge alcohol consumption is more appropriate than another in demonstrating memory deficits in this paradigm, since there is so little previous research related to alcohol and NOR. Multiple schedules of administration could also aide researchers in identifying confounding variables that impact on results such as dosage, periods of withdrawal, and irritation caused by injecting substances. 


\section{Conclusions}

We measured the effect of a binge-like episode of ethanol exposure on performance in both non-spatial and spatial forms of the novel object recognition paradigm in adolescent rats only. This was assessed both 2 days after the final dose of ethanol was administered, and then 1 week later to determine if there was a recovery effect of memory. Rota-rod results during treatment revealed that ethanol treated subjects remained on the rota-rod for significantly less time at two different RPMs compared to saline treated subjects. This indicated that intoxication to the point of impairing motor co-ordination had occurred each day of the five day treatment.

In the NOR task trials, the inter-trial intervals that were implemented between the familiarisation phase (T1) and the testing phase (T2) were limited to a range of between 1 and 5 minutes. This resulted in no significant difference between groups for performance in $\mathrm{T} 2$ of non-spatial and spatial NOR trials in both cycle 1 and cycle 2 of testing. The lack of difference remained consistent when performance was measured by amount of approaches to each object, proportion of approaches to the novel object, exploration time with each object, and proportion of time spent exploring the novel object. As the current results are inconclusive, further investigation is required to determine how appropriate the NOR paradigm is as a measure of ethanol-produced memory impairments. This can potentially be assessed through allowing longer periods of time to explore objects in T1, and by using multiple forms and durations of ethanol administration and comparing and contrasting the subsequent effects. 


\section{References}

Administering Alcohol in Human Studies (2015). Retrieved on March 21, 2016 from www.niaaa.nih.gov/Resources/ResearchResources/job22.htm

Adolescent Health Research Group (2012). The Health and wellbeing of New Zealand secondary school students in 2012. Retrieved March 21, 2016, from https://www.fmhs.auckland.ac.nz/assets/fmhs/faculty/ahrg/docs/2012-overview.pdf

Agartz, I., Momenan, R., Rawlings, R. R., Kerich, M. J., \& Hommer, D. W. (1999). Hippocampal volume in patients with alcohol dependence. Archives of General Psychiatry, 56(4), 356-363.

Alcohol's Damaging Effect on the Brain (2004, October). Retrieved March 14, 2016, from pubs.niaaa.nih.gov/publications/aa63/aa63.htm

Andreollo, N. A., Santos, E. F. D., Araújo, M. R., \& Lopes, L. R. (2012). Rat's age versus human's age: what is the relationship? ABCD. Arquivos Brasileiros de Cirurgia Digestiva (São Paulo), 25(1), 49-51.

Antunes, M., \& Biala, G. (2012). The novel object recognition memory: neurobiology, test procedure, and its modifications. Cognitive processing, 13(2), 93-110.

Barnes, D. E., \& Walker, D. W. (1981). Prenatal Ethanol exposure permanently reduces the number of pyramidal neurons in rat hippocampus. Developmental Brain Research, 1(3), 333-340.

Bava, S., \& Tapert, S. F. (2010). Adolescent brain development and the risk for alcohol and other drug problems. Neuropsychology review, 20(4), 398-413. 
Berry, R. B., \& Matthews, D. B. (2004). Acute Ethanol administration selectively impairs spatial memory in C57BL/6J mice. Alcohol, 32(1), 9-18.

Bird, L. R., Roberts, W. A., Abroms, B., Kit, K. A., \& Crupi, C. (2003). Spatial memory for food hidden by rats (Rattus norvegicus) on the radial maze: studies of memory for where, what, and when. Journal of Comparative Psychology, 117(2), 176.

Broadbent, N. J., Gaskin, S., Squire, L. R., \& Clark, R. E. (2010). Object recognition memory and the rodent hippocampus. Learning \& Memory, 17(1), 5-11.

Burgess, N., Maguire, E. A., \& O'Keefe, J. (2002). The human hippocampus and spatial and episodic memory. Neuron, 35(4), 625-641.

Cagetti, E., Liang, J., Spigelman, I., \& Olsen, R. W. (2003). Withdrawal from chronic intermittent Ethanol treatment changes subunit composition, reduces synaptic function, and decreases behavioral responses to positive allosteric modulators of GABAA receptors. Molecular Pharmacology, 63(1), 53-64.

Camarasa, J., Marimón, J. M., Rodrigo, T., Escubedo, E., \& Pubill, D. (2008). Memantine prevents the cognitive impairment induced by 3, 4-methylenedioxymethamphetamine in rats. European journal of pharmacology, 589(1), 132-139.

Christie, M. M., Bamber, D., Powell, C., Arrindell, T., \& Pant, A. (2013). Older adult problem drinkers: Who presents for alcohol treatment?. Aging \& mental health, 17(1), 24-32.

Chudasama, Y. (2010). Delayed (Non) Match-to-Sample Task. In Encyclopedia of Psychopharmacology (pp. 372-372). Springer Berlin Heidelberg.

Cippitelli, A., Zook, M., Bell, L., Damadzic, R., Eskay, R. L., Schwandt, M., \& Heilig, M. (2010). Reversibility of object recognition but not spatial memory impairment 
following binge-like alcohol exposure in rats. Neurobiology of Learning and Memory, 94(4), 538-546.

Clark, R. E., Zola, S. M., \& Squire, L. R. (2000). Impaired recognition memory in rats after damage to the hippocampus. The Journal of Neuroscience, 20(23), 8853-8860. Chicago

Clements, K. M., Girard, T. A., Ellard, C. G., \& Wainwright, P. E. (2005). Short-Term Memory Impairment and Reduced Hippocampal c-Fos Expression in an Animal Model of Fetal Alcohol Syndrome. Alcoholism: Clinical and Experimental Research, 29(6), 1049-1059.

Cohen, S. J., \& Stackman Jr, R. W. (2014). Assessing rodent hippocampal involvement in the novel object recognition task. A review. Behavioural brain research, 285, 105-117.

De Bellis, M. D., Clark, D. B., Beers, S. R., Soloff, P. H., Boring, A. M., Hall, J., ... \& Keshavan, M. S. (2000). Hippocampal volume in adolescent-onset alcohol use disorders. American Journal of Psychiatry, 157(5), 737-744.

Dlugos, C. A., \& Pentney, R. J. (1997). Morphometric evidence that the total number of synapses on Purkinje neurons of old F344 rats is reduced after long-term Ethanol treatment and restored to control levels after recovery. Alcohol and Alcoholism, 32(2), $161-172$

Drug Facts: The Truth about Alcohol (2015). Retrieved 21 March, 2016 from www.drugfreeworld.org/drugfacts/alcohol/the-youngest-victims.html

Ennaceur, A., \& Delacour, J. (1988). A new one-trial test for neurobiological studies of memory in rats. 1: Behavioral data. Behavioural brain research, 31(1), 47-59. 
Ennaceur, A., \& Meliani, K. (1992). A new one-trial test for neurobiological studies of memory in rats. III. Spatial vs. non-spatial working memory. Behavioural brain research, 51(1), 83-92.

Ennaceur, A., Neave, N., \& Aggleton, J. P. (1997). Spontaneous object recognition and object location memory in rats: the effects of lesions in the cingulate cortices, the medial prefrontal cortex, the cingulum bundle and the fornix. Experimental Brain Research, 113(3), 509-519.

Fact sheet: Alcohol. (2015) Retrieved March 19, 2016, from http://www.who.int/mediacentre/factsheets/fs349/en/

Fact Sheet: Older People's Drinking Habits: Very Little, Very Often. (2013). Retrieved January 2, 2016, from http://www.ias.org.uk/Alcohol-knowledge-centre/Alcohol-andolder-people/Factsheets/Older-peoples-drinking-habits-Very-little-very-often.aspx

Fact Sheets - Alcohol Use and Your Health. (2014, November 7). Retrieved April 22, 2015, from http://www.cdc.gov/alcohol/fact-sheets/alcohol-use.htm

Fact Sheets - Underage Drinking. (2015, November 12). Retrieved December 23, 2015, from http://www.cdc.gov/alcohol/fact-sheets/underage-drinking.htm

García-Moreno, L. M., \& Cimadevilla, J. M. (2012). Acute and chronic Ethanol intake: effects on spatial and non-spatial memory in rats. Alcohol, 46(8), 757-762.

García-Moreno, L. M., Conejo, N. M., Capilla, A., Garcí, O., Senderek, K., \& Arias, J. L. (2002). Chronic Ethanol intake and object recognition in young and adult rats. Progress in Neuro-Psychopharmacology and Biological Psychiatry, 26(5), 831-837.

Giedd, J. N. (2004). Structural magnetic resonance imaging of the adolescent brain. Annals of the New York Academy of Sciences, 1021(1), 77-85. 
Golub, M. S., Hogrefe, C. E., \& VandeVoort, C. A. (2014). Binge drinking prior to pregnancy detection in a nonhuman primate: behavioral evaluation of offspring. Alcoholism: Clinical and Experimental Research, 38(2), 551-556.

Goulart, B. K., de Lima, M. N. M., de Farias, C. B., Reolon, G. K., Almeida, V. R., Quevedo, J., ... \& Roesler, R. (2010). Ketamine impairs recognition memory consolidation and prevents learning-induced increase in hippocampal brain-derived neurotrophic factor levels. Neuroscience, 167(4), 969-973.

Hayes, D. M., Deeny, M. A., Shaner, C. A., \& Nixon, K. (2013). Determining the threshold for alcohol-induced brain damage: New evidence with gliosis markers. Alcoholism: Clinical and Experimental Research, 37(3), 425-434.

Herring, N. R., Schaefer, T. L., Gudelsky, G. A., Vorhees, C. V., \& Williams, M. T. (2008). Effect of (+)-methamphetamine on path integration learning, novel object recognition, and neurotoxicity in rats. Psychopharmacology, 199(4), 637-650.

Hill, A. J. (1978). First occurrence of hippocampal spatial firing in a new environment. Experimental neurology, 62(2), 282-297.

Hiller-Sturmhofel, S., \& Swartzwelder, H. S. (2004). Alcohol's effects on the adolescent brain: what can be learned from animal models. Alcohol Research and Health, 28(4), 213.

Ikonomidou, C., Bittigau, P., Ishimaru, M. J., Wozniak, D. F., Koch, C., Genz, K., ... \& Olney, J. W. (2000). Ethanol-induced apoptotic neurodegeneration and fetal alcohol syndrome. Science, 287(5455), 1056-1060. 
In, H. S., Kim, D. W., Park, Y. M., \& Kim, B. (2014). Experimental intraperitoneal injection of alcohol in rats: Peritoneal findings and histopathology. Toxicology Reports, 1, 3135.

Jensen, G. B., \& Pakkenberg, B. (1993). Do alcoholics drink their neurons away?. The Lancet, 342(8881), 1201-1204.

Kim, C. K., Kalynchuk, L. E., Kornecook, T. J., Mumby, D. G., Dadgar, N. A., Pinel, J. P., \& Weinberg, J. (1997). Object-recognition and spatial learning and memory in rats prenatally exposed to Ethanol. Behavioral neuroscience, 111(5), 985.

King, J. A., Trinkler, I., Hartley, T., Vargha-Khadem, F., \& Burgess, N. (2004). The hippocampal role in spatial memory and the familiarity-recollection distinction: A case study. Neuropsychology, 18(3), 405.

Krazem, A., Marighetto, A., Higueret, P., \& Jaffard, R. (2003). Age-dependent effects of moderate chronic Ethanol administration on different forms of memory expression in mice. Behavioural brain research, 147(1), 17-29.

Kril, J. J., Halliday, G. M., Svoboda, M. D., \& Cartwright, H. (1997). The cerebral cortex is damaged in chronic alcoholics. Neuroscience, 79(4), 983-998.

Lide, D. R. (1994). CRC handbook of chemistry and physics: A ready-reference book of chemical and physical data. Boca Raton, FL: CRC Press.

Logan, R. W., Seggio, J. A., Robinson, S. L., Richard, G. R., \& Rosenwasser, A. M. (2010). Circadian wheel-running activity during withdrawal from chronic intermittent Ethanol exposure in mice. Alcohol, 44(3), 239-244.

McKee, R. D., \& Squire, L. R. (1993). On the development of declarative memory. Journal of Experimental Psychology: Learning, Memory, and Cognition, 19(2), 397. 
McMullen, P. A., Saint-Cyr, J. A., \& Carlen, P. L. (1984). Morphological alterations in rat CA1 hippocampal pyramidal cell dendrites resulting from chronic Ethanol consumption and withdrawal. Journal of comparative neurology, 225(1), 111-118.

Manns, J. R., \& Eichenbaum, H. (2009). A cognitive map for object memory in the hippocampus. Learning \& Memory, 16(10), 616-624.

Manns, J. R., Hopkins, R. O., Reed, J. M., Kitchener, E. G., \& Squire, L. R. (2003). Recognition memory and the human hippocampus. Neuron, 37(1), 171-180.

Manns, J. R., Stark, C. E., \& Squire, L. R. (2000). The visual paired-comparison task as a measure of declarative memory. Proceedings of the National Academy of Sciences, 97(22), 12375-12379.

Markwiese, B. J., Acheson, S. K., Levin, E. D., Wilson, W. A. and Swartzwelder, H. S. (1998), Differential Effects of Ethanol on Memory in Adolescent and Adult Rats. Alcoholism: Clinical and Experimental Research, 22: 416-421.

Matthews, D. B., \& Simson, P. E. (1998). Prenatal exposure to Ethanol disrupts spatial memory: effect of the training-testing delay period. Physiology \& behavior, 64(1), 63-67.

Matthews, D.B., Morrow, A.L., Tokunaga, S., \& McDaniel, J.R. (2002). Acute Ethanol administration and acute allopregnanolone administration impair spatial memory in the Morris water task. Alcoholism: Clinical and Experimental Research, 26, $1747-1751$.

Morris, R. G. M., Garrud, P., Rawlins, J. N. P., \& O'Keefe, J. (1982). Place navigation impaired in rats with hippocampal lesions. Nature, 297(5868), 681-683. 
Morris, R. (1984). Developments of a water-maze procedure for studying spatial learning in the rat. Journal of neuroscience methods, 11(1), 47-60.

Obernier, J.A., White, A.M., Swartzwelder, S.H., \& Crews, F.T. (2002). Cognitive deficits and CNS damage after a 4-day binge Ethanol exposure in rats. Pharmacology, Biochemistry and Behaviour, 72, 521-532.

O'Keefe, J., \& Dostrovsky, J. (1971). The hippocampus as a spatial map. Preliminary evidence from unit activity in the freely-moving rat. Brain research, 34(1), 171-175.

O'keefe, J., \& Nadel, L. (1978). The hippocampus as a cognitive map (Vol. 3, pp. 483-484). Oxford: Clarendon Press.

Oliveira, A. M., Hawk, J. D., Abel, T., \& Havekes, R. (2010). Post-training reversible inactivation of the hippocampus enhances novel object recognition memory. Learning \& Memory, 17(3), 155-160.

Olson, H. C., Feldman, J. J., Streissguth, A. P., Sampson, P. D., \& Bookstein, F. L. (1998). Neuropsychological deficits in adolescents with fetal alcohol syndrome: clinical findings. Alcoholism: Clinical and Experimental Research, 22(9).

Olton, D. S., \& Samuelson, R. J. (1976). Remembrance of places passed: spatial memory in rats. Journal of Experimental Psychology: Animal Behavior Processes, 2(2), 97.

Olton, D. S., Walker, J. A., \& Gage, F. H. (1978). Hippocampal connections and spatial discrimination. Brain research, 139(2), 295-308.

Parker, E. S., Birnbaum, I. M., \& Noble, E. P. (1976). Alcohol and memory: Storage and state dependency. Journal of Verbal Learning and Verbal Behavior, 15(6), 691-702. 
Pascalis, O., \& Bachevalier, J. (1999). Neonatal aspiration lesions of the hippocampal formation impair visual recognition memory when assessed by paired-comparison task but not by delayed nonmatching-to-sample task. Hippocampus, 9(6), 609-616.

Pihlajamäki, M., Tanila, H., Könönen, M., Hänninen, T., Hämäläinen, A., Soininen, H., \& Aronen, H. J. (2004). Visual presentation of novel objects and new spatial arrangements of objects differentially activates the medial temporal lobe subareas in humans. European Journal of Neuroscience, 19(7), 1939-1949.

Pyapali, G. K., Turner, D. A., Wilson, W. A., \& Swartzwelder, H. S. (1999). Age and dosedependent effects of Ethanol on the induction of hippocampal long-term potentiation. Alcohol, 19(2), 107-111.

Rajendran, P., \& Spear, L. P. (2004). The effects of Ethanol on spatial and nonspatial memory in adolescent and adult rats studied using an appetitive paradigm. Annals of the New York Academy of Sciences, 1021(1), 441-444.

Richardson, D. P., Byrnes, M. L., Brien, J. F., Reynolds, J. N., \& Dringenberg, H. C. (2002). Impaired acquisition in the water maze and hippocampal long-term potentiation after chronic prenatal Ethanol exposure in the guinea-pig. European Journal of Neuroscience, 16(8), 1593-1598.

Riley, J. N., \& Walker, D. W. (1978). Morphological alterations in hippocampus after longterm alcohol consumption in mice. Science, 201(4356), 646-648.

Risher, M. L., Fleming, R. L., Risher, W. C., Miller, K. M., Klein, R. C., Wills, T., ... \& Swartzwelder, H. S. (2015). Adolescent Intermittent Alcohol Exposure: Persistence of Structural and Functional Hippocampal Abnormalities into Adulthood. Alcoholism: Clinical and Experimental Research. 
Ryabinin, A. E. (1998). Role of hippocampus in alcohol-induced memory impairment: implications from behavioral and immediate early gene studies. Psychopharmacology, $139(1-2), 34-43$.

Ryabinin, A. E., Criado, J. R., Henriksen, S. J., Bloom, F. E., \& Wilson, M. C. (1997). Differential sensitivity of c-Fos expression in hippocampus and other brain regions to moderate and low doses of alcohol. Molecular psychiatry, 2(1), 32-43.

Ryabinin, A. E., Melia, K. R., Cole, M., Bloom, F. E., \& Wilson, M. C. (1995). Alcohol selectively attenuates stress-induced c-fos expression in rat hippocampus. The Journal of neuroscience, 15(1), 721-730.

Ryabinin, A.E., Miller, M.N., \& Durrant, S. (2002). Effects of acute alcohol administration on object recognition learning in C57BL/6J mice. Pharmacology, Biochemistry and Behaviour, 71, 307-312.

Scaife, J. C., \& Duka, T. (2009). Behavioural measures of frontal lobe function in a population of young social drinkers with binge drinking pattern. Pharmacology Biochemistry and Behavior, 93(3), 354-362.

Schenk, S., Harper, D. N., \& Do, J. (2011). Novel object recognition memory: measurement issues and effects of MDMA self-administration following short inter-trial intervals. Journal of psychopharmacology, 25(8), 1043-1052.

Schindler, A. G., Li, S., \& Chavkin, C. (2010). Behavioral stress may increase the rewarding valence of cocaine-associated cues through a dynorphin/א-opioid receptor-mediated mechanism without affecting associative learning or memory retrieval mechanisms. Neuropsychopharmacology, 35(9), 1932-1942. 
Schröder, N., O'Dell, S. J., \& Marshall, J. F. (2003). Neurotoxic methamphetamine regimen severely impairs recognition memory in rats. Synapse, 49(2), 89-96.

Sircar, R., \& Sircar, D. (2005). Adolescent rats exposed to repeated Ethanol treatment show lingering behavioural impairments. Alcoholism: Clinical and Experimental Research, 29(8), 1402-1410.

Silvers, J. M., Harrod, S. B., Mactutus, C. F., \& Booze, R. M. (2007). Automation of the novel object recognition task for use in adolescent rats. Journal of neuroscience methods, 166(1), 99-103.

Squeglia, L. M., Schweinsburg, A. D., Pulido, C., \& Tapert, S. F. (2011). Adolescent binge drinking linked to abnormal spatial working memory brain activation: differential gender effects. Alcoholism: Clinical and Experimental Research, 35(10), 1831-1841.

Stragier, E., Martin, V., Davenas, E., Poilbout, C., Mongeau, R., Corradetti, R., \& Lanfumey, L. (2015). Brain plasticity and cognitive functions after Ethanol consumption in C57BL/6J mice. Translational psychiatry, 5(12), e696.

Steele, R. J., \& Morris, R. G. M. (1999). Delay-dependent impairment of a matching-to-place task with chronic and intrahippocampal infusion of the NMDA-antagonist D-AP5. Hippocampus, 9(2), 118-136.

Swartzwelder, H. S., Wilson, W. A., \& Tayyeb, M. I. (1995). Age-dependent inhibition of long-term potentiation by Ethanol in immature versus mature Hippocampus. Alcoholism: Clinical and Experimental Research, 19(6), 1480-1485.

Tabakoff, B., \& Hoffman, P. L. (2000). Animal models in alcohol research. Alcohol Research and Health, 24(2), 77-84. 
Tapert, S. F., Brown, G. G., Kindermann, S. S., Cheung, E. H., Frank, L. R., \& Brown, S. A. (2001). fMRI measurement of brain dysfunction in alcohol-dependent young women. Alcoholism: Clinical and Experimental Research, 25(2), 236-245.

Tapert, S. F., Schweinsburg, A. D., Barlett, V. C., Brown, S. A., Frank, L. R., Brown, G. G., \& Meloy, M. J. (2004). Blood oxygen level dependent response and spatial working memory in adolescents with alcohol use disorders. Alcoholism: Clinical and Experimental Research, 28(10), 1577-1586.

Thompson, L. T., \& Best, P. J. (1990). Long-term stability of the place-field activity of single units recorded from the dorsal hippocampus of freely behaving rats. Brain research, 509(2), 299-308.

Townshend, J. M., \& Duka, T. (2005). Binge drinking, cognitive performance and mood in a population of young social drinkers. Alcoholism: Clinical and Experimental Research, 29(3), 317-325.

Uecker, A., \& Nadel, L. (1998). Spatial but not object memory impairments in children with fetal alcohol syndrome. American Journal on Mental Retardation, 103(1), 12-18.

Uzbay, İ. T., \& Kayaalp, S. O. (1995). Heterogeneity of tolerance developed to effects of Ethanol on rotarod and accelerod performances in rats. Alcohol, 12(6), 593-595.

Walker, D. W., Barnes, D. E., Zornetzer, S. F., Hunter, B. E., \& Kubanis, P. (1980). Neuronal loss in hippocampus induced by prolonged Ethanol consumption in rats. Science, 209(4457), 711-713.

Weible, A. P., Rowland, D. C., Pang, R., \& Kentros, C. (2009). Neural correlates of novel object and novel location recognition behavior in the mouse anterior cingulate cortex. Journal of neurophysiology, 102(4), 2055-2068. 
Weissenborn, R., \& Duka, T. (2003). Acute alcohol effects on cognitive function in social drinkers: their relationship to drinking habits. Psychopharmacology, 165(3), 306-312.

Zola, S. M., Squire, L. R., Teng, E., Stefanacci, L., Buffalo, E. A., \& Clark, R. E. (2000). Impaired recognition memory in monkeys after damage limited to the hippocampal region. The Journal of Neuroscience, 20(1), 451-463. 